\title{
Preliminary development, simulation and validation of a weigh in motion system for railway vehicles
}

\section{E. Meli \& L. Pugi}

\section{Meccanica}

An International Journal of Theoretical and Applied Mechanics AIMETA

ISSN 0025-6455

Volume 48

Number 10

Meccanica (2013) 48:2541-2565

DOI 10.1007/s11012-013-9769-9
Meccanica

An International Journal of Theoretical and Applied Mechanics

\section{AIMETA}

\section{包 Springer}


Your article is protected by copyright and all rights are held exclusively by Springer Science +Business Media Dordrecht. This e-offprint is for personal use only and shall not be selfarchived in electronic repositories. If you wish to self-archive your article, please use the accepted manuscript version for posting on your own website. You may further deposit the accepted manuscript version in any repository, provided it is only made publicly available 12 months after official publication or later and provided acknowledgement is given to the original source of publication and a link is inserted to the published article on Springer's website. The link must be accompanied by the following text: "The final publication is available at link.springer.com". 


\title{
Preliminary development, simulation and validation of a weigh in motion system for railway vehicles
}

\author{
E. Meli · L. Pugi
}

Received: 20 November 2012 / Accepted: 25 May 2013 / Published online: 14 June 2013

(C) Springer Science+Business Media Dordrecht 2013

\begin{abstract}
The development of efficient Weigh In Motion (WIM) systems with the aim of estimating the axle loads of railway vehicles in motion is quite interesting both from an industrial and an academic point of view. This kind of systems is very important for safety and maintenance purposes in order to verify the loading conditions of a wide population of vehicles using a limited number of WIM devices distributed on the railway network. The evaluation of the axle load conditions is fundamental especially for freight wagons, more subjected to the risk of unbalanced loads which may be extremely dangerous both for the vehicle safety and the infrastructure maintenance.

In this work the authors present the development, the simulation and the validation of an innovative WIM algorithm with the aim of estimating the axle loads $\widehat{N}$ of railway vehicles (the axle loads include the wheelset weights). The new estimation algorithm is a general purpose one; theoretically it could be applied by considering as input different kinds of track measurements (rail shear, rail bending, sleepers with sensors, etc.) and could be easily customized for different kinds of signals. In the paper a benchmark case based on rail bending measurements is proposed in
\end{abstract}

E. Meli $(\bowtie) \cdot$ L. Pugi

Department of Energy Engineering S. Stecco,

University of Florence, Florence, Italy

e-mail: enrico.meli@unifi.it

L. Pugi

e-mail: luca.pugi@unifi.it which the longitudinal deformations $\varepsilon_{x x}$ measured on the rail foot through strain sensitive elements are used as input. The considered input is affected by noise and bandwidth limitations and, consequently, is a good benchmark to test the robustness of the new algorithm.

To estimate the axle loads, the algorithm approximates the measured physical input through a set of elementary functions calculated by means of a single fictitious load moving on the track. Starting from the set of elementary functions, the measured signal is then reproduced through Least Square Optimization (LSO) techniques: in more detail, the measured signal is considered as a linear combination of the elementary functions, the coefficients of which are the axle loads to be estimated.

Authors have also developed a physical model of the railway track. The model consists of the planar FEM (finite elements method) model of the infrastructure and of the two-dimensional (2D) multibody model of the vehicle (the effects of lateral dynamics are treated as disturbances) and takes into account both the coupling between adjacent loads moving on the track and the vehicle dynamics. The physical model of the track and the innovative WIM algorithm (both considering possible measurement errors) have been validated by means of the experimental data kindly provided by Ansaldo STS and have been implemented in the Matlab and Comsol Multiphysics environments. In particular the model of the railway track has been developed expressly to test the WIM algorithm with a suitable simulation campaign when experimental data 
are not available; in other words it provides simulated inputs to test the WIM algorithm when there are no experimental inputs.

Keywords Railway vehicles - Weigh in motion · Axle load identification

\section{Acronyms:}

WIM: Weigh in motion

FEM: Finite elements method

DOF: Degree of freedom

ODE: Ordinary differential equations

QLH: Quasi-linearity hypothesis

LSO: Least squares optimization

WLSO: Weighted least squares optimization

NLSO: Nonlinear least squares optimization

\section{Introduction}

The development of efficient WIM systems with the aim of estimating the axle loads of railway vehicles in motion is quite interesting both from an industrial and an academic point of view. This kind of systems is very important for safety and maintenance purposes in order to verify the loading conditions of a wide population of vehicles using a limited number of WIM devices distributed on the railway network. The evaluation of the axle load conditions is fundamental especially for freight wagons, more subjected to the risk of unbalanced loads which may be extremely dangerous both for the vehicle safety and the infrastructure maintenance. Some examples of engineering solutions usually employed for WIM applications are shown in Fig. 1:

- rail shear measurements [1-3]: shear stress $\sigma$ and deformation $\varepsilon$ are evaluated on the rail by means of a circular notch/slot drilled over the rail web on the mean-neutral line where approximately pure shear stresses are present. Stress-deformations concentrations arising in the notch are measured using conventional strain gage sensors or piezoelectric/piezo-resistive systems in order to estimate the rail shear efforts and consequently the vertical force $\widehat{N}$ due to axle load.

- rail bending measurements [4, 5]: strain sensitive elements are used to evaluate stress $\sigma$ and deformation $\varepsilon$ due to the bending (typically on the rail foot) and then to estimate the vertical load $\widehat{N}$. Contactless measurements may be also performed using laser Doppler sensors.

- sleepers with sensors [6]: force sensitive elements placed over the sleepers in the section corresponding to the rail baseplate/pads allow the measurement of the force on the sleeper $N_{S}$ and, starting from that, the estimation of the axle load $\widehat{N}$.

- sensitive bridge/slab track systems [7]: in this solution sleepers and rails are constrained over an instrumented structure which works as spring element permitting the measurement of the force $N_{S}$ on the structure and thus the estimation of the axle load $\widehat{N}$.

WIM systems based on rail stress $\sigma$ and deformation $\varepsilon$ measurements (rail shear and bending measurements) are the most diffused mainly since they usually assure higher performances in terms of bandwidth, precision and linearity. Moreover, with respect to other measurement solutions, they are less affected by different construction methods of the infrastructure (ballasted, with slab track, etc.).

Both shear and bending measurements on the rail may be optimized in order to reject as much as possible spurious signals due to the lateral forces and to the longitudinal tensile components coming from the thermally induced coaction loads. Lateral dynamics of the vehicle may also produce load transfers between the left and right wheels of the same axle. In order to measure the total load on each axle, the stress and deformation measurements on the left and right sides of the same measurement section should be averaged.

In this work the authors present the development, the simulation and the validation of an innovative WIM algorithm with the aim of estimating the axle loads $\widehat{N}$ of railway vehicles (especially freight wagons); the weights of the wheelsets are included into the loads $\widehat{N}$. The estimation algorithm proposed in this work can be applied to a generic set of input signals derived from track measurements (rail shear, rail bending, sleepers with sensors, etc.) because it is not designed for a specific sensor layout. The benchmark case studied in this paper is based on rail bending measurements performed through strain sensitive elements used to evaluate the longitudinal deformations $\varepsilon_{x x}$ on the rail foot mainly for two reasons:

- availability of experimental data: thanks to the cooperation with our industrial partner (Ansaldo STS); 


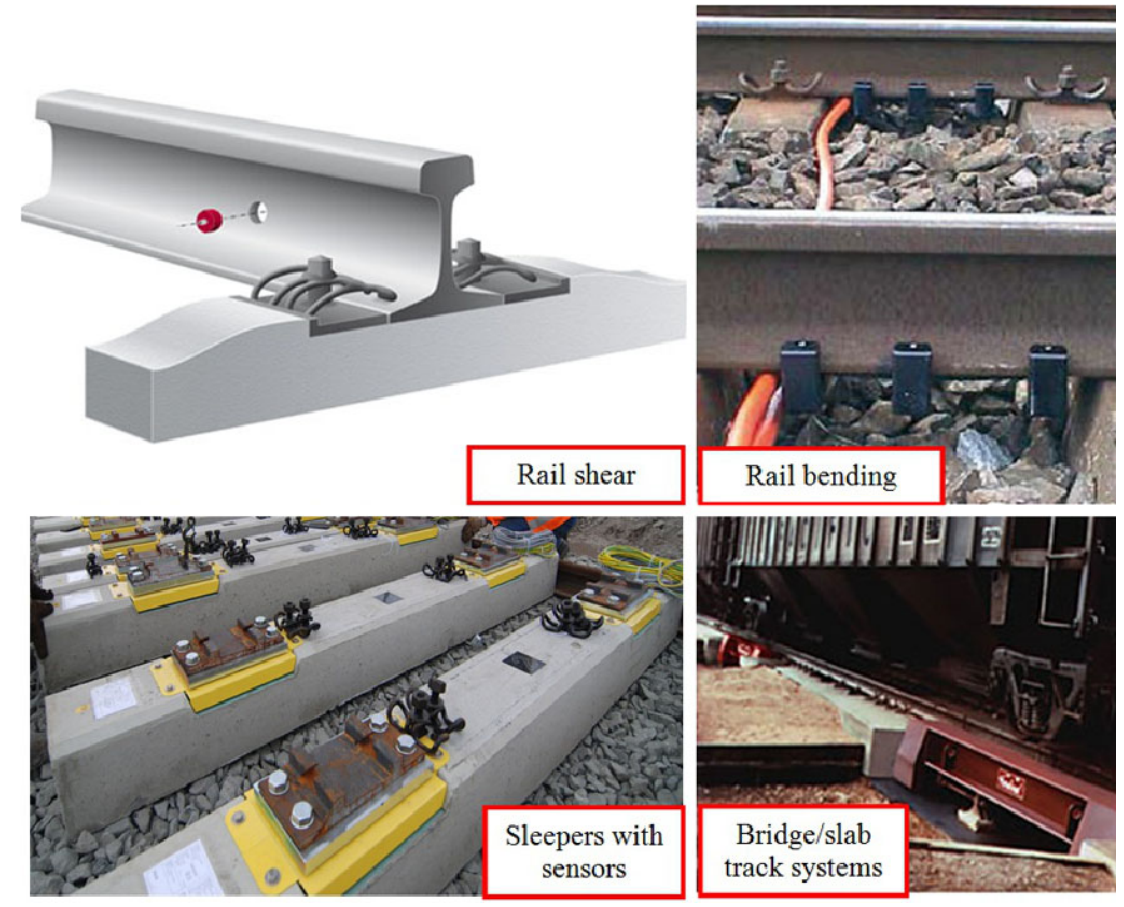

Fig. 1 Engineering solutions for WIM applications

- verification of algorithm robustness: bending measurements are often affected by noise and bandwidth limitations (if compared to shear measurements) and consequently the chosen input is a good benchmark to verify the robustness of the proposed approach.

To estimate the axle loads, the algorithm approximates the measured physical input (in this case the longitudinal deformation) through a set of elementary functions (a basis of functions, mathematically speaking). To evaluate the elementary functions, a single fictitious moving load is simulated (see Fig. 15). This elementary model has necessary to be very simple and depend on few significant parameters that can be easily tuned, for example, by performing the estimation on a known train travelling through the measure station. In particular the simple model for the basis construction cannot depend on the vehicle geometrical and physical parameters, obviously unknown. Starting from the set of elementary functions, the measured signal is then approximated by means of Least Square Optimization (LSO) techniques: in more detail, the measured signal is considered as a linear combination of the elementary functions, the coefficients of which are the axle loads to be estimated [20-22].
Finally it is worth noting that the new WIM algorithm can be quite useful not only to estimate the axle loads $\widehat{N}$ but also to different purposes like the control and the prediction of the axle loads during the design process of railway vehicles. This interesting feature of the procedure is mainly due to general structure of the algorithm and, at the same time, to its simplicity.

\section{General architecture of the system}

The general architecture of the system is schematically shown in Fig. 2. The main element of the whole system is the innovative WIM algorithm for the estimation of the vertical axle loads $\widehat{N}$ on railway vehicles (especially freight wagons); the weights of the wheelsets are included into the loads $\widehat{N}$. The algorithm is based on rail bending measurements performed through strain sensitive elements used to evaluate the longitudinal deformations on the rail foot. These deformations (simulated $\varepsilon_{x x}$ if provided by a physical model of the railway track or real $\varepsilon_{x x}^{s p}$ if coming from experimental data; see Fig. 2) represent the physical inputs of the WIM algorithm that, starting from the knowledge of these physical quantities, estimates the axle loads $\widehat{N}$. 


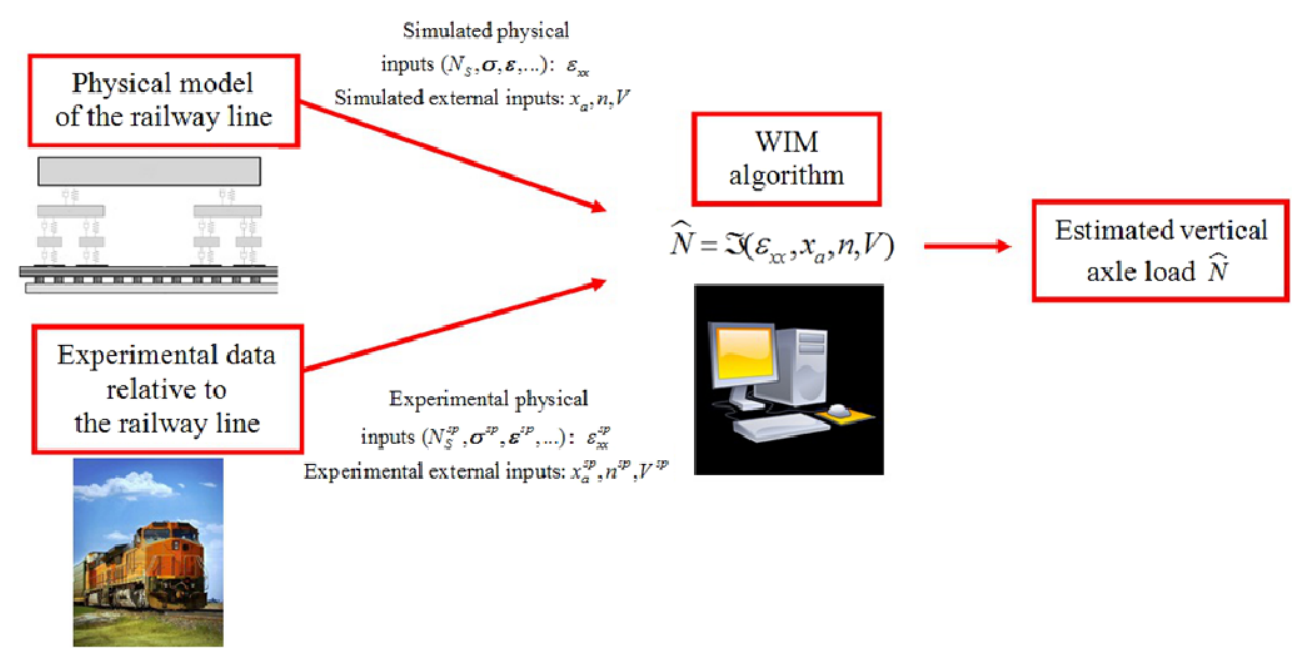

Fig. 2 General architecture of the system

Besides the longitudinal deformations on the rail foot $\varepsilon_{x x}$, the WIM algorithm also needs some additional information (external inputs) concerning the vehicle speed, the axle number and the axle positions inside the railway vehicle $\left(V, n, x_{a}\right.$ in case of simulated external inputs and $V^{s p}, n^{s p}, x_{a}^{s p}$ in case of experimental external inputs; see Fig. 2). These further physical quantities can be identified using by example additional sensors or transmitted by the vehicle itself using low cost technologies.

The WIM system consists of various measure points (few if possible to reduce both the measure station dimensions and the economic costs) distributed along the railway track on the rail foot between two contiguous sleepers to amplify the longitudinal deformations $\varepsilon_{x x}$. On both the sides of the track measure points are present to reject the effect of spurious signals and of the load transfers produced by the lateral dynamics (see Fig. 3). The innovative architecture of the WIM algorithm allows also the exploitation of different inputs like generic stresses $\sigma, \sigma^{s p}$ and deformations $\varepsilon, \varepsilon^{s p}$ and the forces on the sleepers $N_{S}, N_{S}^{s p}$ (both simulated and experimental) as well as a combination of such physical quantities.

During the research activity, the authors have also employed a physical model of the whole railway track. Since the measurement layout is designed to reject the effects of lateral dynamics as disturbances, all the system is studied using simplified planar models of the track. In particular the railway track model consists of a planar FEM model of the infrastructure and of a 2D

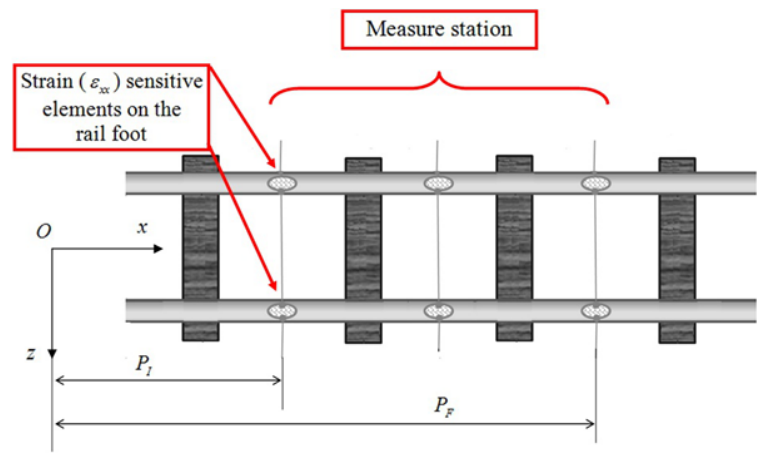

Fig. 3 Measure station: a possible layout of the measure points

multibody model of the vehicle and takes into account both the coupling between the adjacent loads moving on the track and the vehicle dynamics (see Figs. 4 and 8). The physical model of the track and the innovative WIM algorithm (both considering possible measurement errors) have been then validated by means of the experimental data kindly provided by Ansaldo STS [19] and have been implemented in the Matlab and Comsol Multiphysics environments [8, 18]. In the study case the model of the railway track has been developed expressly to test the WIM algorithm with a suitable simulation campaign when experimental data are not available; in other words it provides simulated inputs to test the WIM algorithm when there are no experimental inputs. Obviously this model is not used inside the WIM algorithm because the geometrical and physical train parameters are unknown. 


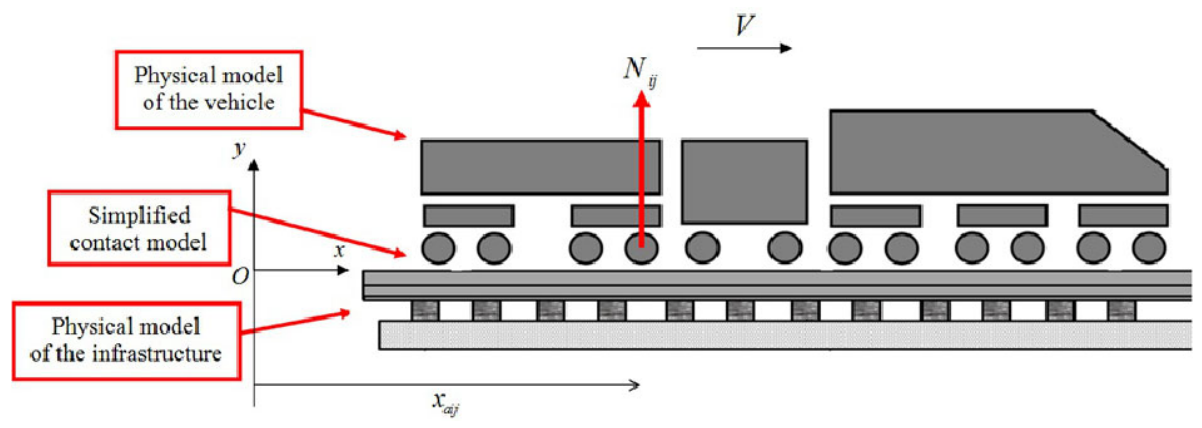

Fig. 4 The physical model of the railway track

\section{Physical model of the railway track}

The physical model of the railway track is briefly described in Fig. 4. Since the effects of the lateral dynamics are treated as disturbances, the whole model consists of a planar FEM model of the infrastructure (rail, sleepers and ballast), a 2D multibody model of the vehicle and a simplified contact model between the vehicle wheels and the rails. In the rest of the paper $x_{a i j}$ will indicate the initial position of the $i$ th axle of the $j$ th wagon of the vehicle while $N_{i j}$ will be the generic vertical axle loads, the estimate $\widehat{N}_{i j}$ of which will be evaluated by the presented WIM algorithm; the weights of the wheelsets are included into the loads $\widehat{N}_{i j}, N_{i j}$. As previously said, the longitudinal deformations have to be averaged on the left and right rails before being considered as input of the WIM algorithm; coherently also the generic vertical axle loads $N_{i j}$ and their estimations $\widehat{N}_{i j}$ will indicate the average loads on the left and right wheels. Anyway the proposed approach can be easily used both for axle and wheel loads measurements. In fact, in case of wheel load estimation, a similar procedure can be applied by considering $\widehat{N}_{i j}, N_{i j}$ as the vertical loads on the single wheels (without average).

\subsection{The infrastructure model}

Rail and underlying infrastructures are modelled as a continuous beam representing the rail supported by an elastic foundation which simulates sleepers and ballast. For the rail both Eulero-Bernoulli and RaleighTimoshenko models can be used [9]. In particular the Eulero-Bernoulli beam model neglects the shear deformability considering only the contribution of the

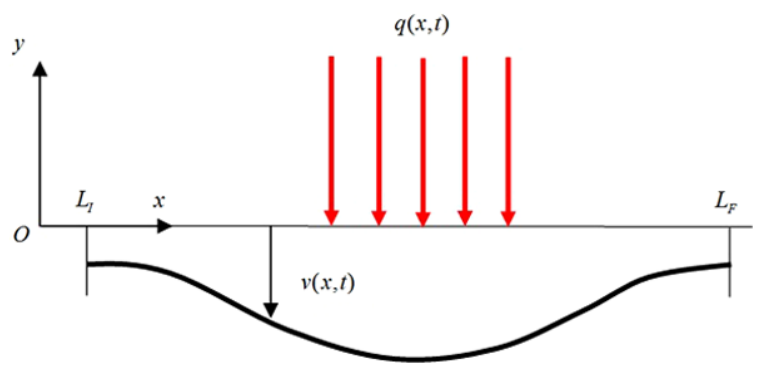

Fig. 5 Beam model of the rail

bending (see Fig. 5):

$E I \frac{\partial^{4} v(x, t)}{\partial x^{4}}+\rho A \frac{\partial^{2} v(x, t)}{\partial t^{2}}=q(x, t)$

where $x \in\left[L_{I}, L_{F}\right]$ is the longitudinal abscissa, $t \in\left[T_{I}, T_{F}\right]$ is the time, $E$ and $\rho$ are the Young modulus and the density of the beam, $A$ and $I$ are the area and the momentum of the beam section, $q(x, t)$ is the distributed load and $v(x, t)$ is the vertical displacement. The initial conditions associated to Eq. (1) are $v\left(x, T_{I}\right)=0 \forall x \in\left[L_{I}, L_{F}\right]$ and $\frac{\partial v}{\partial t}\left(x, T_{I}\right)=0$ $\forall x \in\left[L_{I}, L_{F}\right]$ respectively while there are no boundary conditions because the beam is connected only to the sleepers as it will be better clarified in the following sections. The contribution of shear deformability mainly affects higher frequency modes where the Eulero-Bernoulli model is more likely to overestimate the corresponding eigen-frequencies. Since the bandwidth of the real system (infrastructure and measurement chain) is unknown and anyway much lower than $100 \mathrm{~Hz}$, authors prefer to use the EuleroBernoulli model to simulate the rails because it assures a good accuracy up to $100-200 \mathrm{~Hz}$ in terms bandwidth (frequencies much higher than the characteristic bandwidth of the whole system in realistic condi- 
Table 1 Main characteristics of the rail beam model

\begin{tabular}{lll}
\hline Parameter & Units & Value \\
\hline Young modulus $E$ & $\mathrm{~Pa}$ & $2.1 * 10^{11}$ \\
Density $\rho$ & $\mathrm{kg} / \mathrm{m}^{3}$ & $7.8 * 10^{3}$ \\
Area of the beam section $A$ & $\mathrm{~m}^{2}$ & $7.686 * 10^{-3}$ \\
Momentum of the beam section $I$ & $\mathrm{~m}^{4}$ & $3.055 * 10^{-5}$ \\
Rayleigh damping coefficient $\alpha_{r}$ & $\mathrm{~s}^{-1}$ & 30 \\
$\begin{array}{l}\text { Rayleigh damping coefficient } \beta_{r} \\
\text { Distance between neutral section } \\
\text { and rail foot } y_{f}\end{array}$ & $\mathrm{~s}$ & 0.003 \\
\hline
\end{tabular}

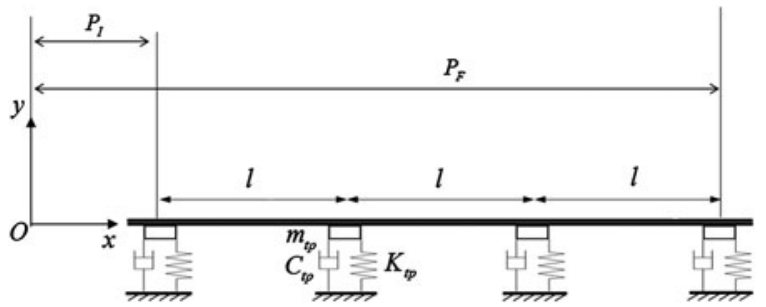

Fig. 6 The Kisilowski model of the sleepers-ballast ensemble

tions); moreover higher frequency modes are heavily affected by damping and by other non-linear phenomena that would have to be calibrated on experimental data $[9,10,13-17]$. In particular in this work the structural damping of the rail is modelled using the socalled "proportional" or Rayleigh damping; the damping matrix $\mathbf{C}$ is calculated as a linear combination of the inertia $\mathbf{M}$ and stiffness $\mathbf{K}$ matrices of the structure:

$\mathbf{C}=\alpha_{r} \mathbf{M}+\beta_{r} \mathbf{K}$

where the matrices $\mathbf{M}$ and $\mathbf{K}$ derive from the FEM discretization of the rail beam. The coefficients $\alpha_{r}$ and $\beta_{r}$ are calibrated in order to fit the typical behaviour expected from experimental results and physical considerations available in literature $[9,13,17]$.

The main physical characteristics of the rail beam model are reported in Table 1; in this work the UIC60 rail profile has been adopted.

The stiffness and damping constants associated to the $p$ th sleepers-ballast system are $K_{t p}$ and $C_{t p}$ while $m_{t p}$ are the concentrated masses (see Fig. 6). The longitudinal position $x_{t p}$ of the $p$ th $1 \mathrm{DOF}$ system modelling the sleepers-ballast ensemble and the vertical position $y_{t p}$ of the $p$ th mass $m_{t}$ rigidly connected to
Table 2 Main characteristics of the sleepers-ballast system

\begin{tabular}{lll}
\hline Parameter & Units & Value \\
\hline $\begin{array}{l}\text { Lumped mass associated to the } \\
\text { sleeper-ballast system } m_{t}\end{array}$ & $\mathrm{~kg}$ & 10 \\
$\begin{array}{l}\text { Stiffness associated to the } \\
\text { sleepers-ballast system } K_{t}\end{array}$ & $\mathrm{~N} / \mathrm{m}$ & $5 * 10^{7}$ \\
$\begin{array}{l}\text { Damping associated to the } \\
\text { sleepers-ballast system } C_{t}\end{array}$ & $\mathrm{Ns} / \mathrm{m}$ & $2.5 * 10^{5}$ \\
$\begin{array}{l}\text { Sleepers distance } l \\
\text { Sleepers total number } N_{t}\end{array}$ & $\mathrm{~m}$ & 0.6 \\
$\begin{array}{l}\text { Beginning of the track } L_{I} \\
\text { End of the track } L_{F}\end{array}$ & - & 201 \\
\hline
\end{tabular}

the rail beam can be expressed as follows:

$$
\begin{gathered}
x_{t p}=L_{I}+(p-1) l, \quad y_{t p}(t)=v\left(x_{t p}, t\right), \\
p=1,2, \ldots, N_{t}
\end{gathered}
$$

where $x_{t 1}=L_{I}, x_{t N_{t}}=L_{F}\left(L_{I}\right.$ and $L_{F}$ are the beginning and the end of the track respectively), $l$ is the distance between two contiguous sleepers and $N_{t}$ is the total number of sleepers. Table 2 summarizes the main physical quantities relative to the sleepers-ballast system.

In order to model the contribution of the deformability of sleepers and ballast different models of increasing complexity may be adopted. One of the simplest formulations may be the so called "Winkler continuous supported beam" $[10,24,25]$ that models ballast and sleepers as a continuous visco-elastic soil to which the rail is constrained. The Winkler model neglects the discrete nature of the sleepers-ballast system and introduces approximations that are too large for the study of a WIM algorithm. To consider the discrete nature of the sleepers-ballast ensemble, the sleepers have to be modelled as lumped systems of springs, masses and dampers which try to reproduce/fit the modal behaviour of the infrastructure. Increasing the number of degrees of freedom used to model sleepers and ballast usually leads to an improvement of the model accuracy. In particular one of the most accurate models is introduced by Dahlberg [9, 13].

For the simulation and the validation of the proposed WIM algorithm authors preferred to use the simplified model proposed by Kisilowski [11] which is often used in literature for the simulation and the development of this kind of applications [1, 2, 12]. In the considered model the sleepers-ballast ensemble is 
simulated by means of single degree of freedom systems resulting a good compromise between accuracy and efficiency (see Fig. 6). The lumped parameters associated to the sleepers-ballast system and described in Table 2 (such as the lumped mass $m_{t}$, the stiffness $K_{t}$ and the damping $C_{t}$ of the sleeper-ballast system) do not represent any physical property (like the real mass of the sleepers) but are simple modal-numerical parameters that have to be tuned to obtain the desired modal and dynamical behaviour of the sleeper-ballast system.

As regards the longitudinal deformations on the rail foot $\varepsilon_{x x}$ representing the main physical inputs of the WIM algorithm, they are measured on various measure points (few if possible to reduce both the measure station dimensions and the economic costs) distributed along the railway track and placed between two contiguous sleepers to amplify the longitudinal deformations (see Figs. 3 and 7). On both the sides of the track measure points are present to reject the effect of spurious signals and of the load transfers produced by the lateral dynamics. The longitudinal position $x_{m k}$ of the $k$ th couple of measure points and the mean value $\varepsilon_{x x k}$ of longitudinal deformation measured in those points will be

$$
\begin{aligned}
x_{m k} & =P_{I}+(k-1) l, \quad \varepsilon_{x x k}(t)=\varepsilon\left(x_{m k}, t\right), \\
k & =1,2, \ldots, N_{m}
\end{aligned}
$$

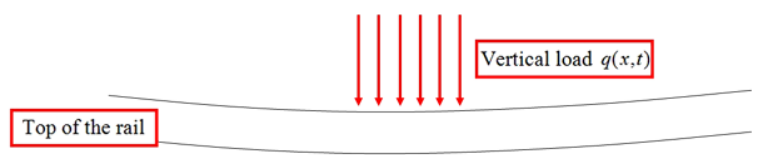

Foot of the rail

Strain $\left(\varepsilon_{\mathrm{xx}}\right)$ sensitive elements

Fig. 7 Measurement of the longitudinal deformations $\varepsilon_{x x}$ on the rail foot between two contiguous sleepers where $x_{m 1}=P_{I}$ and $x_{t N_{m}}=P_{F}$ are the positions of the initial and final points of the measure station (obviously placed exactly between two contiguous sleepers) and $N_{m}$ is the total number of measure points. It has to be noticed that the measure points $x_{m k}$ may also not be consecutive; however they always have to be placed between two contiguous sleepers. In this case the first part of Eq. (4) becomes $x_{m k}=P_{I}+(k-1) l_{k}$ where $l_{k}$ (a multiple of $l$ ) is the distance between two consecutive sleepers.

Finally, according to the Eulero-Bernoulli beam model, the longitudinal deformations $\varepsilon_{x x k}$ in each measure point can be calculated starting from the knowledge of the vertical displacement $v(x, t)$ :

$\varepsilon_{x x k}(t)=-y_{f} \frac{\partial^{2} v\left(x_{m k}, t\right)}{\partial x^{2}}$

where $y_{f}<0$ is the distance of rail foot from the neutral line of the rail section.

\subsection{The vehicle model}

The benchmark train configuration adopted in this paper is briefly described in Fig. 4 and in Table 3. The planar 2D multibody model considered by the authors is illustrated in Fig. 8; for the sake of simplicity only the third wagon is described.

The degrees of freedom (DOF) taken into account in the model include the vertical displacements of all the bodies $\left(y_{c j}, y_{b i j}, y_{w i j}\right)$ and the pitch angles of carbody and bogies $\left(\varphi_{c j}, \varphi_{b i j}\right)$. The inertial properties of carbody, bodies and wheels are respectively $m_{c j} I_{c j}, m_{b i j} I_{b i j}$ and $m_{w i j} I_{w i j}$ while $K_{s i j} C_{s i j}, K_{p i j}$ $C_{p i j}$ and $K_{c i j} C_{c i j}$ indicate the stiffness and damping constants of the suspension stages and of the contact model between wheels and rails. Since the longitudinal deformations $\varepsilon_{x x k}$ and the vertical axle loads $N_{i j}$ are averaged on the left and right rails and on the left and right wheels respectively, for symmetry reasons only one half of the body masses and inertial tensors

Table 3 Benchmark train composition

\begin{tabular}{lllllll}
\hline Vehicle & Wheelset & Prim. susp. & Sec. susp. & Axle load (t) & Bogie dist. (m) & Wheelbase (m) \\
\hline Locomotive & b-b-b & yes & yes & 17.7 & 5.25 & 2.15 \\
Second wagon & $1-1$ & yes & no & 8.0 & - & 9 \\
Third wagon & $2-2$ & yes & yes & 7.8 & 15.8 & 1.8 \\
\hline
\end{tabular}




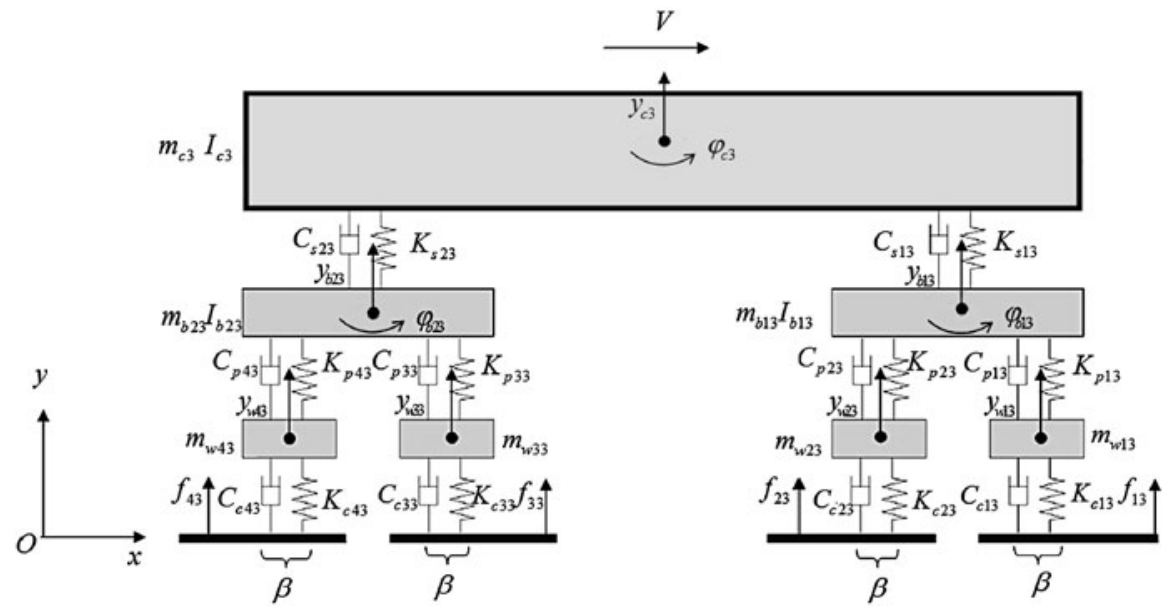

Fig. 8 Complete 2D multibody model of the railway vehicle

has to be considered while the stiffness and damping constants have not to be modified.

The simplified contact model is based on the hypotheses that wheel and rail interact to each other on a contact segment of length $\beta$ (having the same magnitude order of the contact patch longitudinal axis) and that the vertical contact load $q_{i j}(x, t)$ is uniformly distributed on the contact segment (see Figs. 8 and 9).

Starting from the position of the generic train axle $x_{i j}=x_{a i j}+t V(V$ is the longitudinal train speed supposed to be constant within the measure station), the mean vertical displacement $f_{i j}$ on the contact segment $\beta$ correspondent to the axle $x_{i j}$ can be evaluated as follows

$f_{i j}(t)=\frac{1}{\beta} \int_{L_{i}}^{L_{f}} v(x, t) o q\left(\frac{x-x_{i j}}{\beta}\right) d x$

where the square wave $o q$ is defined as

$o q(u)= \begin{cases}1 & -1 / 2 \leq u \leq 1 / 2, \\ 0 & \text { otherwise }\end{cases}$

At this point it is possible to evaluate both the vertical load on the rail beam $q_{i j}$ and the vertical force on the train wheel $F_{i j}$ :

$$
\begin{aligned}
& q_{i j}(x, t) \\
& =\frac{1}{\beta}\left[K_{c i j}\left(y_{w i j}-f_{i j}-r_{i j}\right)+C_{c i j}\left(\dot{y}_{w i j}-\dot{f}_{i j}\right)\right] \\
& \quad \times o q\left(\frac{x-x_{i j}}{\beta}\right)
\end{aligned}
$$

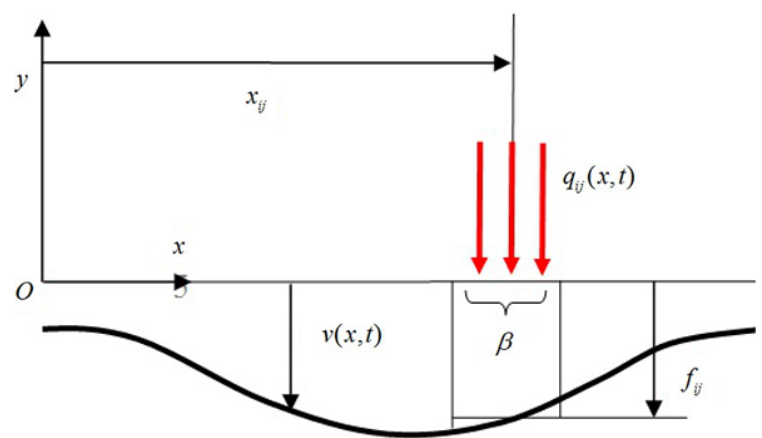

Fig. 9 Simplified contact model

$$
\begin{aligned}
& F_{i j}(t) \\
& \quad=-\left[K_{c i j}\left(y_{w i j}-f_{i j}-r_{i j}\right)+C_{c i j}\left(\dot{y}_{w i j}-\dot{f}_{i j}\right)\right]
\end{aligned}
$$

where $y_{w i j}(t)$ is the vertical displacement of the center of mass of the axle $x_{i j}$ and $r_{i j}$ is the wheel radius.

In Table 4 the main characteristics of the vehicle model are reported in terms of inertial quantities, suspensions data, geometry, nominal total loads and considered speed range while Table 5 summarizes the main contact characteristics at the wheel-rail interface.

\subsection{Measurement errors}

In order to improve the accuracy of the physical model of the railway track (infrastructure model and vehicle model) fhe following disturbances have been considered: 
Table 4 Main characteristics of the vehicle model

\begin{tabular}{|c|c|c|}
\hline Parameter & Units & Value \\
\hline Axle numbers $n_{t o t}, n, n_{1}, n_{2}, n_{3}$ & - & $12,3,6,2,4$ \\
\hline Axle init. positions (locomotive: $x_{a 11}, \ldots, x_{a 61}$ ) & $\mathrm{m}$ & $52.15,50,46.9,44.75,41.65,39.5$ \\
\hline Axle init. positions (second wagon: $x_{a 12}, x_{a 22}$ ) & $\mathrm{m}$ & $33.8,24.8$ \\
\hline Axle init. positions (third wagon: $x_{a 13}, \ldots, x_{a 43}$ ) & $\mathrm{m}$ & $19.6,17.8,3.8,2$ \\
\hline Inertial prop. (loc. car body: $m_{c 1}, I_{c 1}$ ) & $\mathrm{kg}, \mathrm{kg} \mathrm{m}^{2}$ & 84400,1125000 \\
\hline Inertial prop. (loc. bogies: $m_{b 11}, I_{b 11}, \ldots, m_{b 31}, I_{b 31}$ ) & $\mathrm{kg}, \mathrm{kg} \mathrm{m}^{2}$ & 3000,3100 \\
\hline Inertial prop. (loc. wheelsets: $m_{w 11}, I_{w 11}, \ldots, m_{w 61}, I_{w 61}$ ) & $\mathrm{kg}, \mathrm{kg} \mathrm{m}^{2}$ & 2100,120 \\
\hline Inertial prop. (2nd wagon car body: $m_{c 2}, I_{c 2}$ ) & $\mathrm{kg}, \mathrm{kg} \mathrm{m}^{2}$ & 12400,84000 \\
\hline Inertial prop. (2nd wagon wheelsets: $m_{w 12}, I_{w 12}, m_{w 22}, I_{w 22}$ ) & $\mathrm{kg}, \mathrm{kg} \mathrm{m}^{2}$ & 1800,112 \\
\hline Inertial prop. (3rd wagon car body: $m_{c 3}, I_{c 3}$ ) & $\mathrm{kg}, \mathrm{kg} \mathrm{m}^{2}$ & 18600,480000 \\
\hline Inertial prop. (3rd wagon bogies: $m_{b 13}, I_{b 13}, m_{b 23}, I_{b 23}$ ) & $\mathrm{kg}, \mathrm{kg} \mathrm{m}^{2}$ & 2600,1500 \\
\hline Inertial prop. (3rd wagon wheelsets: $m_{w 13}, I_{w 13}, \ldots, m_{w 43}, I_{w 43}$ ) & $\mathrm{kg}, \mathrm{kg} \mathrm{m}^{2}$ & 1800,112 \\
\hline Prop. of primary susp. (loc.: $K_{p 11}, C_{p 11}, \ldots, K_{p 61}, C_{p 61}$ ) & $\mathrm{N} / \mathrm{m}, \mathrm{Ns} / \mathrm{m}$ & 2050000,7000 \\
\hline Prop. of secondary susp. (loc.: $\left.K_{s 11}, C_{s 11}, \ldots, K_{s 31}, C_{s 31}\right)$ & $\mathrm{N} / \mathrm{m}, \mathrm{Ns} / \mathrm{m}$ & 710000,50000 \\
\hline Prop. of primary susp. (2nd wagon: $K_{p 12}, C_{p 12}, K_{p 22}, C_{p 22}$ ) & $\mathrm{N} / \mathrm{m}, \mathrm{Ns} / \mathrm{m}$ & 815000,4000 \\
\hline Prop. of primary susp. (3rd wagon: $K_{p 13}, C_{p 13}, \ldots, K_{p 43}, C_{p 43}$ ) & $\mathrm{N} / \mathrm{m}, \mathrm{Ns} / \mathrm{m}$ & 780000,3000 \\
\hline Prop. of secondary susp. (3rd wagon: $K_{s 13}, C_{s 13}, K_{s 23}, C_{s 23}$ ) & $\mathrm{N} / \mathrm{m}, \mathrm{Ns} / \mathrm{m}$ & 235000,18000 \\
\hline Wheel radius (loc.: $\left.r_{11}, \ldots, r_{61}\right)$ & $\mathrm{m}$ & 0.625 \\
\hline Wheel radius (2nd wagon: $r_{12}, r_{22}$ ) & $\mathrm{m}$ & 0.46 \\
\hline Wheel radius (3rd wagon: $r_{13}, \ldots, r_{43}$ ) & $\mathrm{m}$ & 0.46 \\
\hline Nom. total loads on the wheels (locomotive: $N_{11}, \ldots, N_{61}$ ) & $\mathrm{N}$ & 86655 \\
\hline Nom. total loads on the wheels (second wagon: $N_{12}, N_{22}$ ) & $\mathrm{N}$ & 39240 \\
\hline Nom. total loads on the wheels (locomotive: $N_{13}, \ldots, N_{43}$ ) & $\mathrm{N}$ & 38014 \\
\hline Considered speed range for freight trains $V$ & $\mathrm{~m} / \mathrm{s}$ & $10-40$ \\
\hline
\end{tabular}

Table 5 Main contact characteristics at the wheel-rail interface

\begin{tabular}{lll}
\hline Parameter & Units & Value \\
\hline Contact stiffnesses $K_{c 11}, \ldots, K_{c 61}, K_{c 12}, K_{c 22}, K_{c 13}, \ldots, K_{c 43}$ & $\mathrm{~N} / \mathrm{m}$ & $1 * 10^{8}$ \\
Contact dampings $C_{c 11}, \ldots, C_{c 61}, C_{c 12}, C_{c 22}, C_{c 13}, \ldots, C_{c 43}$ & $\mathrm{Ns} / \mathrm{m}$ & 45000 \\
Contact segment $\beta$ & $\mathrm{m}$ & 0.01 \\
\hline
\end{tabular}

- numerical disturbances and bias errors on the longitudinal axle positions $x_{i j}$ : the imperfect knowledge of the positions $x_{i j}$ is simulated by adding a suitable numerical noise to the original physical quantity

$x_{i j}^{r}(t)=x_{i j}(t)+U_{x i j}\left[\mu_{x}, \delta_{x} / 2\right]$

where $U_{x i j}$ is a uniform distribution of mean $\mu_{x}$ and amplitude $\delta_{x}$.
- frequency effects on the signal $\varepsilon_{x x k}$ due to the limited band of physical system and measurement chain: the frequency effects due to the limited band of the real system and the rail measurement chain have been modelled through a second order low pass filter directly applied to the considered physical signal $\varepsilon_{x x k}$

$\varepsilon_{x x k}^{f}(t)=B_{2, \omega_{n}}(s) \varepsilon_{x x k}(t)$ 
Table 6 Disturbances and errors

\begin{tabular}{lll}
\hline Parameter & Units & Value \\
\hline $\begin{array}{l}\text { Mean of the disturbance on the } \\
\text { longitudinal axle positions } \mu_{x}\end{array}$ & $\mathrm{~m}$ & 0.001 \\
$\begin{array}{l}\text { Amplitude of the disturbance on } \\
\text { the longitudinal axle positions } \delta_{x}\end{array}$ & $\mathrm{~m}$ & 0.01 \\
$\begin{array}{l}\text { Mean of the disturbance on the } \\
\text { longitudinal deformations } \mu_{\varepsilon}\end{array}$ & strain & $1 * 10^{-6}$ \\
$\begin{array}{l}\text { Amplitude of the disturbance on } \\
\text { the longitudinal deformations } \delta_{\varepsilon}\end{array}$ & strain & $2.5 * 10^{-5}$ \\
$\begin{array}{l}\text { Cut frequency considered range } \\
f_{n} \text { of the filter }\end{array}$ & $\mathrm{Hz}$ & $10-40$ \\
\hline
\end{tabular}

where $B_{2, \omega_{n}}(s)$ is the second order Butterworth filter and $\omega_{n}=2 \pi f_{n}$ is the cut frequency $\left(\omega_{n}\right.$ in $\mathrm{rad} / \mathrm{s}$ and $f_{n}$ in $\mathrm{Hz}$ ).

- numerical disturbances and bias errors on the signal $\varepsilon_{x x k}^{f}$ : besides the frequency effects, also numerical disturbances and bias errors on the signal $\varepsilon_{x x k}^{f}$ have been modelled

$\varepsilon_{x x k}^{f r}(t)=\varepsilon_{x x k}^{f}(t)+U_{\varepsilon k}\left[\mu_{\varepsilon}, \delta_{\varepsilon} / 2\right]$

where this time $\mu_{\varepsilon}$ and $\delta_{\varepsilon}$ are the mean and the amplitude of the disturbance distribution $U_{\varepsilon k}$. The aim of numerical disturbances and bias errors on the signal $\varepsilon_{x x k}^{f}$ is to properly reproduce the numerical noise affecting the measurement; therefore they have to be applied to the signal only after the low pass filter.

The values of the disturbances taken into account are visible in Table 6 together with the cut frequency considered range of the filter.

By way of example the comparison between the original deformation $\varepsilon_{x x 1}(t)=\varepsilon_{x x}\left(x_{m 1}, t\right)$ and the deformation $\varepsilon_{x x 1}^{f r}(t)=\varepsilon_{x x}^{f r}\left(x_{m 1}, t\right)$ taking into account the numerical disturbances (both evaluated on the single measure point $x_{m 1}=60.3 \mathrm{~m}$ ) is shown in Fig. 10 . The simulation has been performed at a speed of $V=50 \mathrm{~km} / \mathrm{h}\left(T_{I}=0, T_{F}=5 \mathrm{~s}\right)$ with the vehicle multibody model (see Sect. 3.2) while the cut frequency of the system is equal to $f_{n}=20 \mathrm{~Hz}$.

In Fig. 10 the numerical disturbances both on the longitudinal axle positions $x_{i j}^{r}(t)$ and on the deformation $\varepsilon_{x x 1}^{f r}(t)$ are visible. Moreover the time history of $\varepsilon_{x x 1}^{f r}(t)$ also highlights the frequency effects due to the limited band of the physical system in terms of signal amplitude and phase.
In the following of the paper the measurement errors will be not considered during the model and WIM algorithm validation (see Sects. 3.4 and 5.1) because of the high quality of the experimental data and the low train speed $V$. However the measurement errors will play a fundamental role when the physical model of the railway track will be employed to test the accuracy and the robustness of the WIM algorithm in absence of experimental data (Section 5.2).

\subsection{Validation of the railway track model}

The physical model of the railway track (infrastructure model and vehicle model) has been validated by means of experimental data provided by Ansaldo STS [19]. In particular the experimental data consist in the longitudinal deformations on the rail foot $\varepsilon_{x x 1}^{s p}(t)=\varepsilon_{x x}^{s p}\left(x_{m 1}, t\right)$ and $\varepsilon_{x x 2}^{s p}(t)=\varepsilon_{x x}^{s p}\left(x_{m 2}, t\right)$ measured in two measure points $x_{m 1}=P_{I}=60.3 \mathrm{~m}$, $x_{m 2}=P_{F}=65.1 \mathrm{~m}$ (with $N_{m}=2$ ). The experimental data have been compared with the analogous quantities $\varepsilon_{x x 1}(t)=\varepsilon_{x x}\left(x_{m 1}, t\right), \varepsilon_{x x 2}(t)=\varepsilon_{x x}\left(x_{m 2}, t\right)$ simulated through the railway track model (in the benchmark case $V=15.4 \mathrm{~km} / \mathrm{h}, T_{I}=0 \mathrm{~s}, T_{F}=15.3 \mathrm{~s}$ ).

Figures 11 and 12 show the comparison between the measured deformations $\varepsilon_{x x 1}^{s p}, \varepsilon_{x x 2}^{s p}$ and the simulated ones $\varepsilon_{x x 1}, \varepsilon_{x x 2}$ in the measure points $x_{m 1}, x_{m 2}$. As visible in the figures, the comparison highlights a good agreement between experimental and simulated quantities.

In particular, considering a general comparison between the global shape of the simulated solution and the corresponding experimental behavior, the matching is encouraging; on the other hand higher errors are clearly appreciable on the peak values. This is a very interesting feature considering that the aim of this work is to demonstrate the robustness of the proposed approach with respect to this kind of errors and disturbances.

The numerical results have been obtained by using the variable order and variable step ODE integrator ODE15s (specifically designed for stiffness problems) for the time integration; the algorithm PARDISO has been employed to solve the linear systems arising form the FEM discretization of the rail beam. Table 7 summarizes the values of the main parameters of the ODE integrator like the maximum step size MaxStep, the absolute and relative tolerances AbsTol, RelTol and the maximum dimension $h_{\max }$ of the elements used in the FEM discretization of the rail beam [20-22]. 


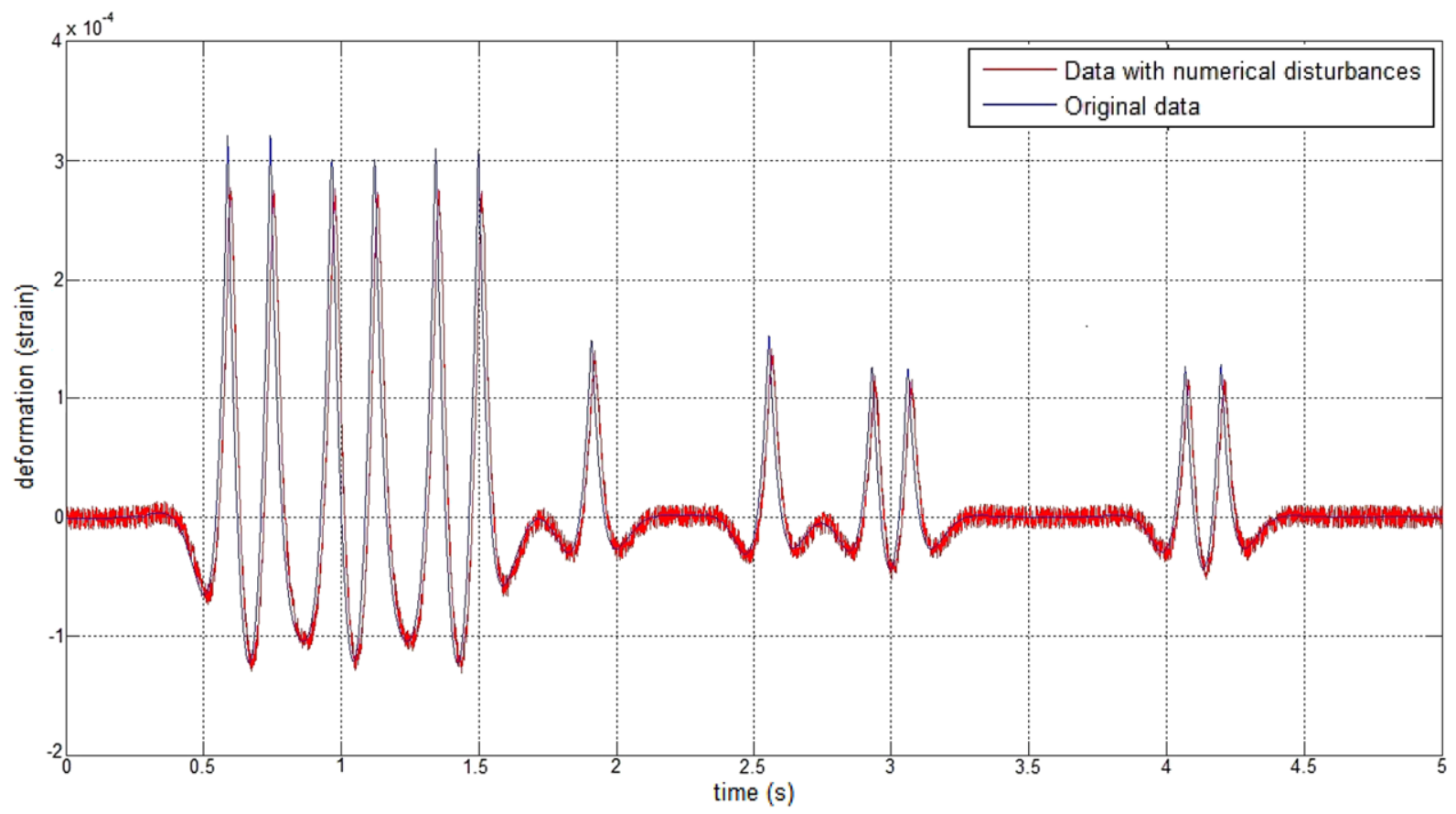

Fig. 10 Original longitudinal deformation $\varepsilon_{x x 1}(t)$ and longitudinal deformation $\varepsilon_{x x 1}^{f r}(t)$ with numerical disturbances in the measure point $x_{m 1}=60.3 \mathrm{~m}$ (cut frequency equal to $f_{n}=20 \mathrm{~Hz}$ )

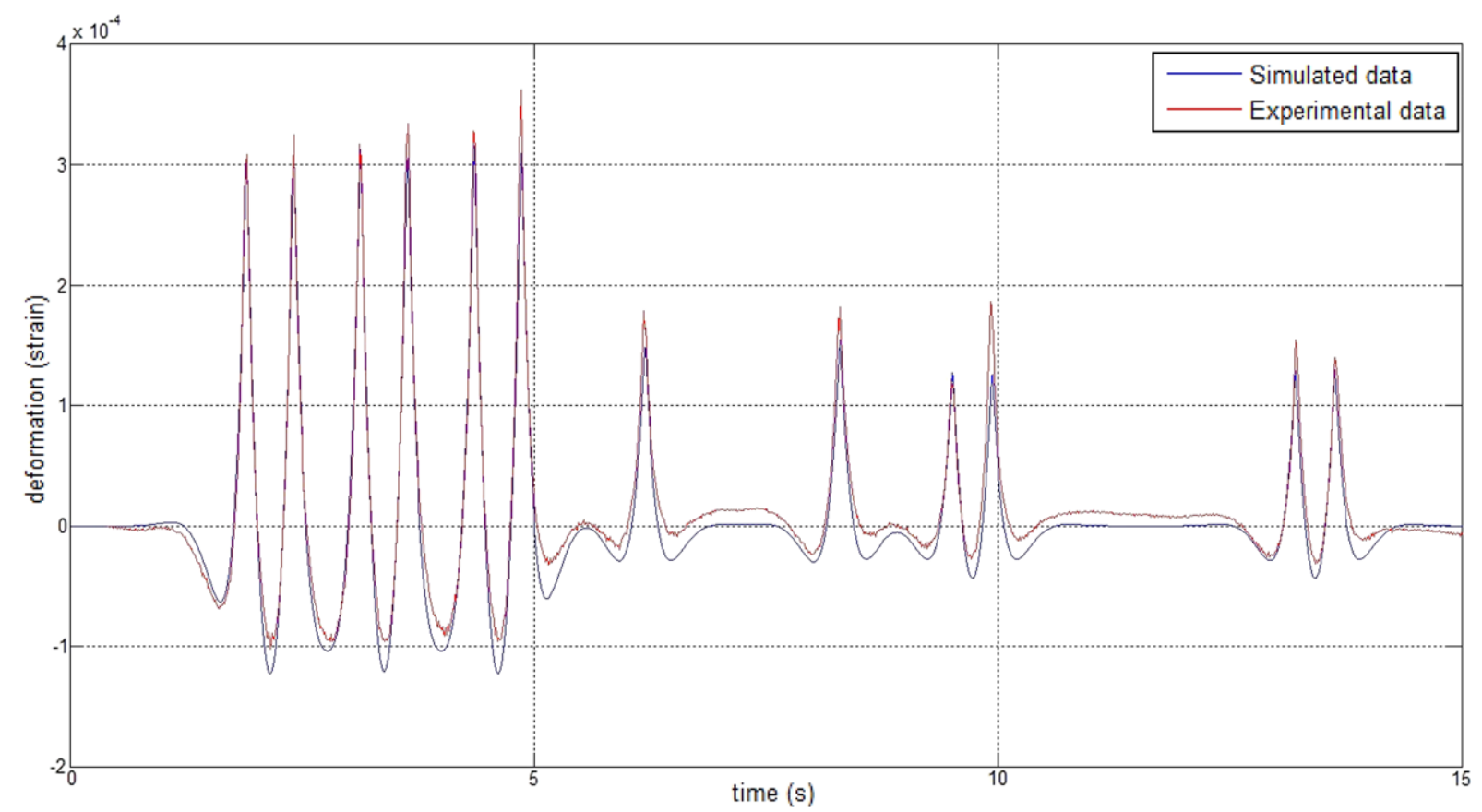

Fig. 11 Measured and simulated longitudinal deformations $\varepsilon_{x x 1}^{s p}$ and $\varepsilon_{x x 1}$ in the measure point $x_{m 1}=60.3 \mathrm{~m}$ 


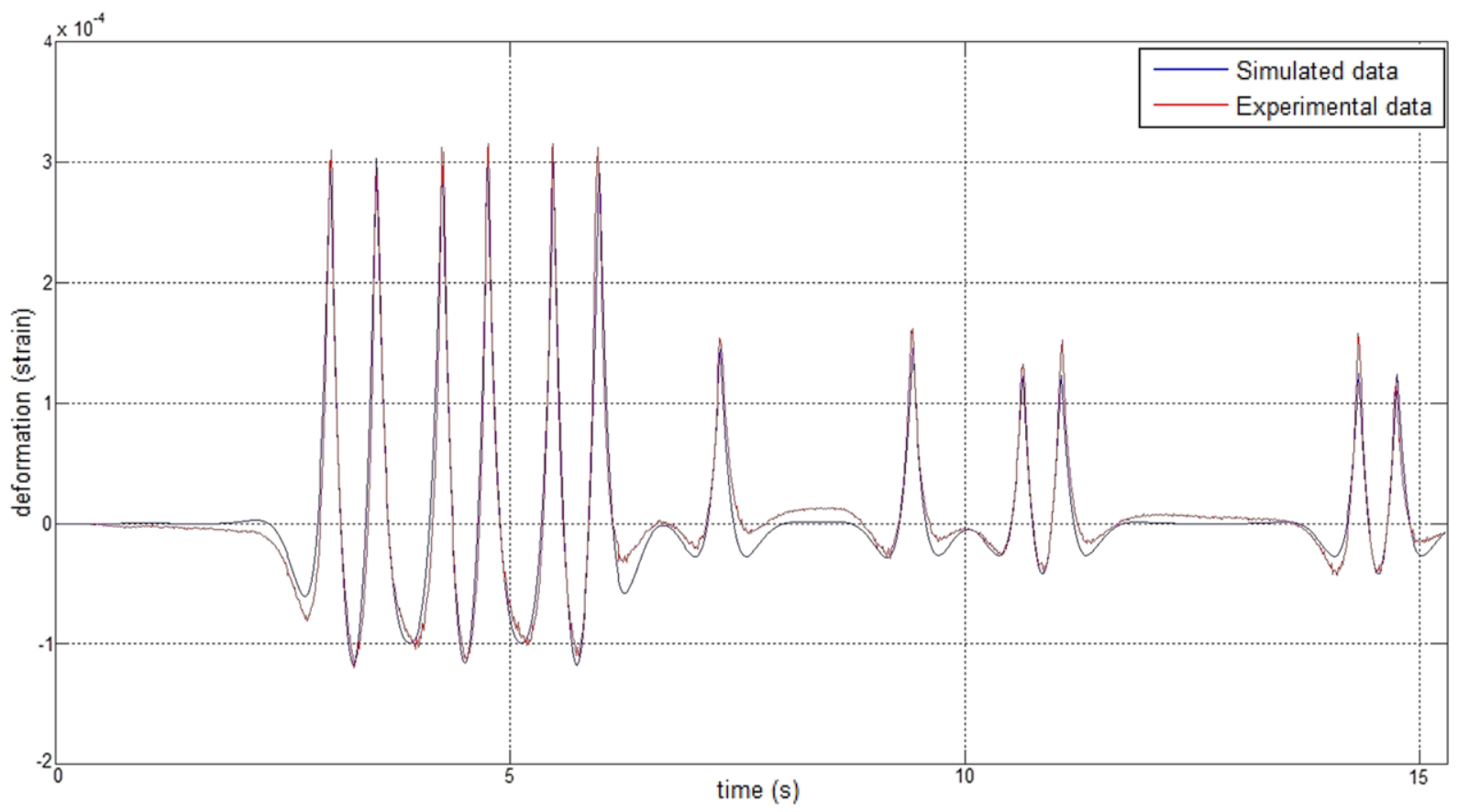

Fig. 12 Measured and simulated longitudinal deformations $\varepsilon_{x x 2}^{s p}$ and $\varepsilon_{x x 2}$ in the measure point $x_{m 2}=65.1 \mathrm{~m}$

Table 7 Main parameters of the ODE integrator

\begin{tabular}{lll}
\hline Parameter & Units & Value \\
\hline MaxStep & $\mathrm{s}$ & $10^{-4}$ \\
AbsTol & - & $10^{-5}$ \\
RelTol & - & $10^{-6}$ \\
$h_{\max }$ & $\mathrm{m}$ & 0.001 \\
\hline
\end{tabular}

\section{WIM algorithm}

In this section the innovative WIM algorithm for the estimation of the vertical axle loads $\widehat{N}$ on railway vehicles is described (the weights of the wheelsets are included into the loads $\widehat{N}$ ).

\subsection{Architecture of the WIM algorithm}

The general architecture of the system is schematically reported in the diagram in Fig. 13.

The developed WIM algorithm evaluates the estimation $\widehat{N}_{i j}$ of the vertical axle loads $N_{i j}$ starting from the knowledge of all the deformations $\varepsilon_{x x k}^{f r}$ relative to the measure points $x_{m k}$ of the measure station; in absence of numerical disturbances (circumstance indicated by authors) the deformations $\varepsilon_{x x k}^{f r}$ will be equal to the original signal $\varepsilon_{x x k}$. Moreover the WIM algorithm also needs some additional information (external inputs) concerning the vehicle speed $V$, the axle number $n$ and the axle positions inside the railway vehicle $x_{a i j}$. These further physical quantities can be identified using by example additional sensors or transmitted by the vehicle itself using low cost technologies. Obviously the WIM algorithm can work both with synthetic inputs provided by the numerical model (see Sect. 3.2) and with experimental data directly measured on the railway track; in this second case the algorithm inputs $\left(\varepsilon_{x x k}^{s p}, V^{s p}, n^{s p}\right.$, and $\left.x_{a}^{s p}\right)$ will be marked with the apex $s p$.

The main idea behind the new WIM algorithm consists in approximating the measured physical input (in this case the longitudinal deformation) through a set of elementary functions (a basis of functions). To evaluate the elementary functions a single fictitious load $N_{f}$ moving on the track is simulated (see Figs. 14 and 15).

This elementary model must be very simple and depend on few significant parameters that can be easily tuned (for example by performing the estimation on a known train travelling through the measure station). In particular the simple model for the basis construction cannot depend on the vehicle geometrical and physical parameters, obviously unknown. 
Because of the global structure of the physical signals considered as inputs, it is quite intuitive to suppose the system approximatively linear with respect to the vertical loads $N_{i j}$ (the so-called quasi-linearity hypothesis (QLH)); in other words the effect of the generic load $N_{i j}$ on the longitudinal deformations $\varepsilon_{x x k}^{f r}$ is assumed not to be affected by the presence of the other loads (especially the contiguous ones).

Under this assumption, by applying the superposition of effects principle, it is possible to estimate both the simulated longitudinal deformations $\varepsilon_{x x k}^{f r}$ and the experimental ones $\varepsilon_{x x k}^{s p}$ produced by the whole train through a linear combination of $n_{\text {tot }}$ deformations $\varepsilon_{x x k i j}$ produced by $n_{\text {tot }}$ single fictitious loads $N_{f}$ (one for each vehicle axle) properly shifted in the time of a delay $t_{i j}$ (Section 4.2). A schematic simplified illustration of the quasi-linearity hypothesis is reported in Fig. 14. The elementary deformations $\varepsilon_{x x k i j}$ represent the set of elementary functions (the basis of functions) needed to approximate the original physical inputs $\left(\varepsilon_{x x k}^{f r}\right.$ or $\left.\varepsilon_{x x k}^{s p}\right)$. In this case the linear combination coefficients are equal to $\widehat{N}_{i j} / N_{f}$.

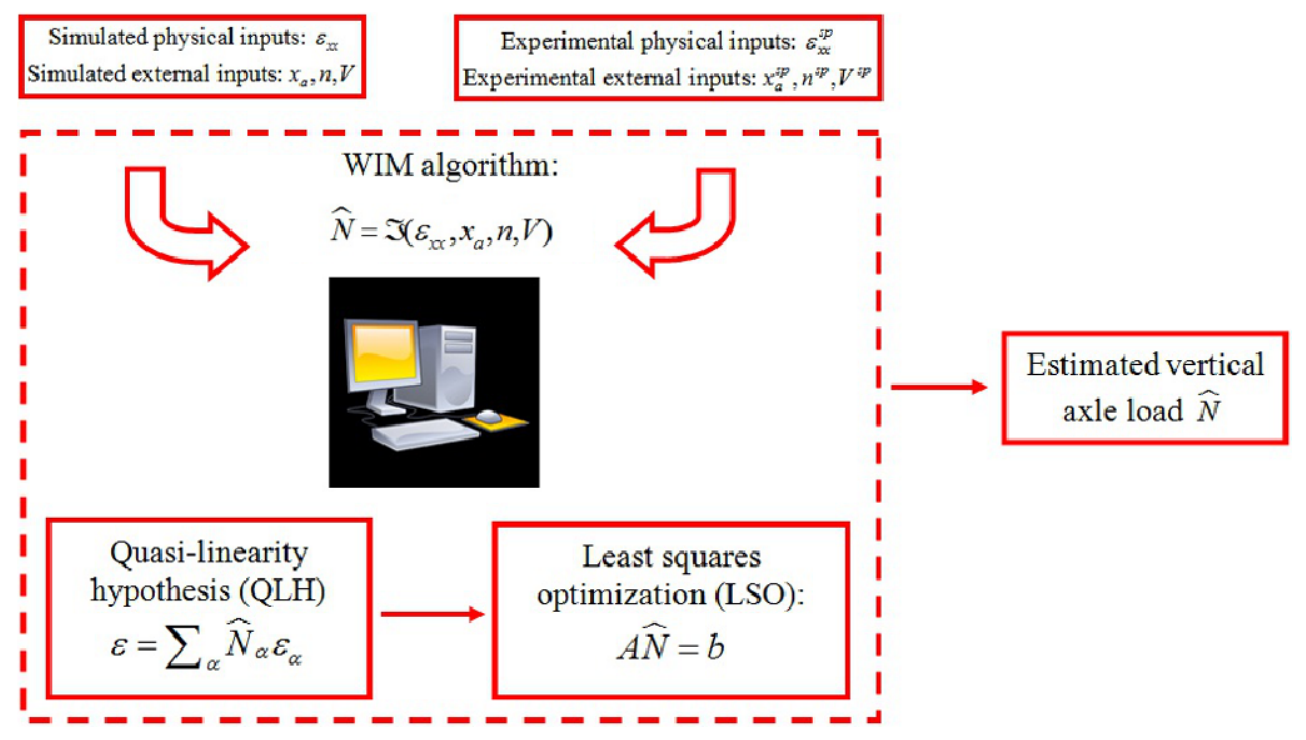

Fig. 13 Architecture of the WIM algorithm
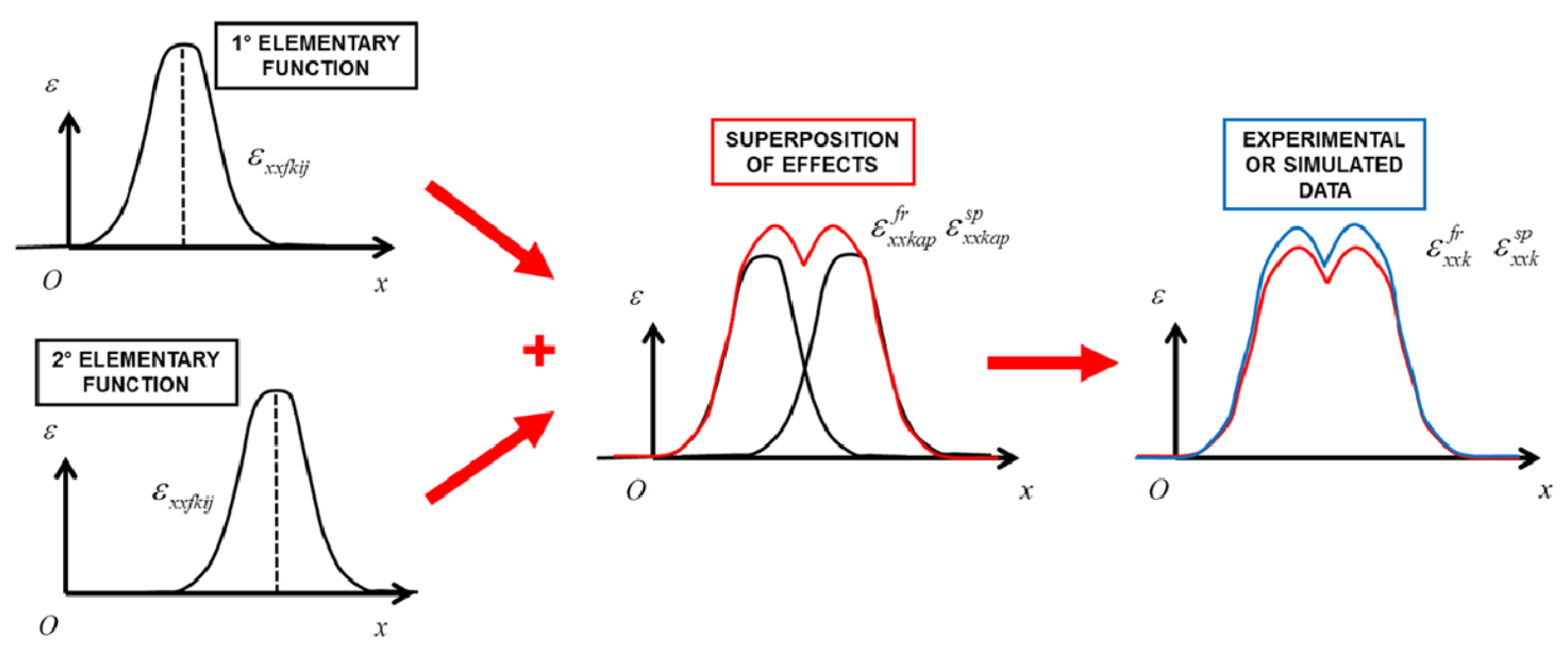

Fig. 14 Schematic representation of the quasi-linearity hypothesis (QLH) 


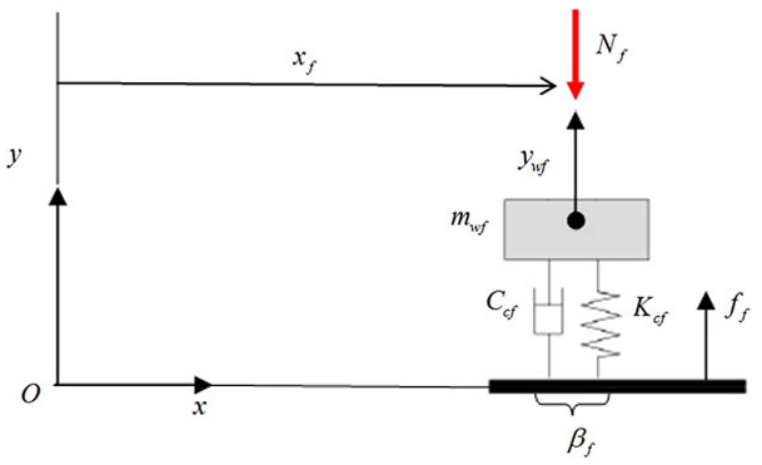

Fig. 15 Fictitious load

Table 8 Fictitious contact characteristics at the wheel-rail interface

\begin{tabular}{lll}
\hline Parameter & Units & Value \\
\hline Contact stiffness $K_{f}$ & $\mathrm{~N} / \mathrm{m}$ & $1 * 10^{8}$ \\
Contact damping $C_{f}$ & $\mathrm{Ns} / \mathrm{m}$ & 45000 \\
Contact segment $\beta_{f}$ & $\mathrm{~m}$ & 0.01 \\
Wheelset mass $m_{w f}$ & $\mathrm{~kg}$ & 2000 \\
Fictitious load $N_{f}$ & $\mathrm{~N}$ & 65000 \\
\hline
\end{tabular}

Evidently, in order to correctly apply the superposition of effects principle, the quasi-linearity hypothesis (QLH) must hold within the whole range of velocities $V$ and cut frequencies $f_{n}$ considered for freight trains.

Finally, since the system can be considered only approximately linear, a least squares optimization (LSO) is needed to minimize the approximation error and, at the same time, to optimize the values of $\widehat{N}_{i j}$.

\subsection{The quasi-linearity hypothesis}

As previously stated, if the quasi-linearity hypothesis holds, by applying the superposition of effects principle it is possible to estimate both the simulated longitudinal deformations $\varepsilon_{x x k}^{f r}$ and the experimental ones $\varepsilon_{x x k}^{s p}$ produced by the whole train through a linear combination of $n_{t o t}$ deformations $\varepsilon_{x x k i j}$ produced by $n_{t o t}$ single fictitious loads $N_{f}$ (one for each vehicle axle) properly shifted in the time of a delay $t_{i j}$. The fictitious contact variables are reported in Fig. 15 and Table 8 (only one half of the fictitious mass $m_{w f}$ has to be considered).

The adopted values of the fictitious contact variables are of first attempt and have to be optimized to improve the accuracy of the WIM algorithm. To properly highlight how the whole WIM algorithm could be
Table 9 Tuning parameters of the WIM algorithm

\begin{tabular}{lll}
\hline Parameter & Units & Value \\
\hline $\begin{array}{l}\text { Lumped mass associated to the } \\
\text { sleeper-ballast system } m_{t}\end{array}$ & $\mathrm{~kg}$ & 10 \\
$\begin{array}{l}\text { Stiffness associated to the } \\
\text { sleepers-ballast system } K_{t}\end{array}$ & $\mathrm{~N} / \mathrm{m}$ & $5 * 10^{7}$ \\
$\begin{array}{l}\text { Damping associated to the } \\
\text { sleepers-ballast system } C_{t}\end{array}$ & $\mathrm{Ns} / \mathrm{m}$ & $2.5 * 10^{5}$ \\
$\begin{array}{l}\text { Contact stiffness } K_{f} \\
\text { Contact damping } C_{f}\end{array}$ & $\mathrm{~N} / \mathrm{m}$ & $1 * 10^{8}$ \\
Wheelset mass $m_{w f}$ & $\mathrm{Ns} / \mathrm{m}$ & 45000 \\
\hline
\end{tabular}

easily tuned, in Table 9 all the tuning parameters of the elementary model are summarized.

As previously said, this elementary model has necessary to be very simple and depend on few physical parameters that can be easily estimated during the tuning process (for example, by performing the estimation on a known benchmark train travelling through the measure station). In this case the tuning parameters comprise the lumped quantities associated to the sleeper-ballast system $m_{t}, K_{t}, C_{t}$ (see Sect. 3.1) and the fictitious parameters $m_{w f}, K_{f}, C_{f}$. All the previous quantities can be easily optimized to obtain a good approximation of the desired modal and dynamical behaviour of the system.

In particular the position of the fictitious load $N_{f}$ along the track and the relative longitudinal deformation $\varepsilon_{x x f k}$ are defined as

$\varepsilon_{x x f k}(t)=\varepsilon_{x x f}\left(x_{m k}, t\right), \quad x_{f}=x_{a f}+t * V$

where $x_{a f}=0 \mathrm{~m}$ and $t \in\left[T_{I}, T_{F}\right]$. In this way the $n_{t o t}$ deformations $\varepsilon_{x x k i j}$ produced by the $n_{t o t}$ single fictitious loads $N_{f}$ and their positions $x_{f i j}$ can be evaluated by introducing suitable time delays $t_{i j}$

$t_{i j}=\frac{x_{a i j}-x_{a f}}{V}$

and by applying such delays to the deformation $\varepsilon_{x x f k}$ and the position $x_{f}$ :

$\varepsilon_{x x f k i j}(t)=\varepsilon_{x x f k}\left(t-t_{i j}\right)$,

$x_{f i j}=x_{a f}+\left(t+t_{i j}\right) * V=x_{a i j}+t * V=x_{i j}$

where $t \in\left[T_{I}, T_{F}-t_{i j}\right]$. At this point, thanks to the superposition of effects principle, both the simulated longitudinal deformations $\varepsilon_{x x k}^{f r}$ and the experimental 


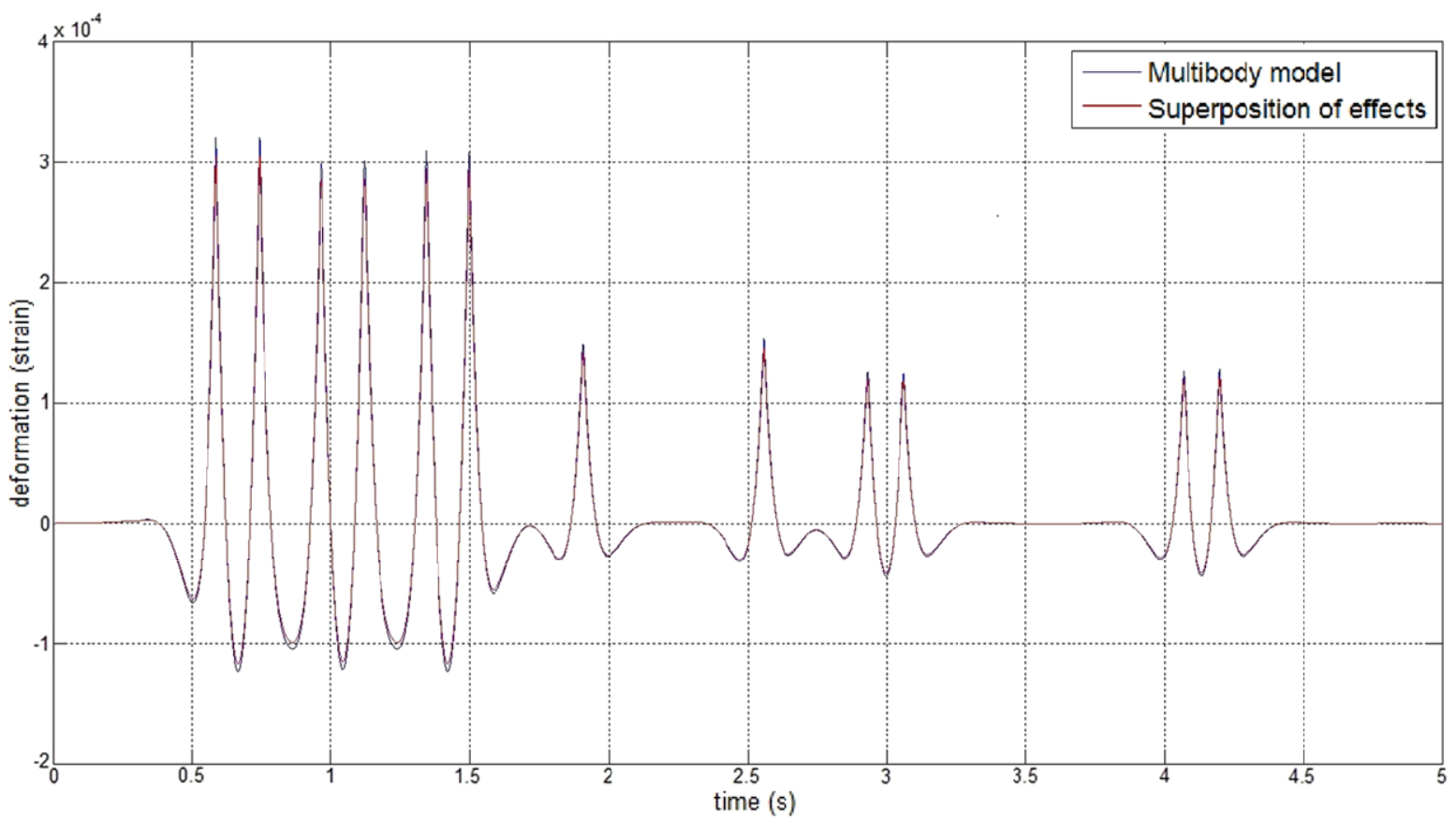

Fig. 16 Comparison between the deformations obtained with the multibody vehicle model $\varepsilon_{x x 1}^{f r}(t)$ and with the fictitious vertical loads $\varepsilon_{x x 1 a p}^{f r}(t)$ according to the quasi-linearity hypothesis

ones $\varepsilon_{x x k}^{s p}$ produced by the whole train can be approximated as follows:

$$
\begin{gathered}
\varepsilon_{x x k}^{f r}(t) \simeq \varepsilon_{x x k a p}^{f r}(t)=\sum_{j=i}^{n} \sum_{i=1}^{n_{j}} \alpha_{i j}^{s i m} \varepsilon_{x x f k i j}(t), \\
\alpha_{i j}^{s i m}=\frac{\hat{N}_{i j}^{s i m}}{N_{f}}, \quad k=1,2, \ldots, N_{m} \\
\varepsilon_{x x k}^{s p}(t) \simeq \varepsilon_{x x k a p}^{s p}(t)=\sum_{j=i}^{n} \sum_{i=1}^{n_{j}} \alpha_{i j}^{s p} \varepsilon_{x x f k i j}(t), \\
\alpha_{i j}^{s p}=\frac{\hat{N}_{i j}^{s p}}{N_{f}}, \quad k=1,2, \ldots, N_{m}
\end{gathered}
$$

where, as said before, the linear combination coefficients $\alpha_{i j}^{s i m}, \alpha_{i j}^{s p}$ are proportional to the estimated vertical loads $\widehat{N}_{i j}^{s i m}$ and $\widehat{N}_{i j}^{m i s}$.

By way of example, to confirm the validity of the quasi-linearity hypothesis (see Fig. 14), a comparison between the results obtained with the multibody vehicle model (see Sect. 3.2) and those obtained by means of fictitious vertical loads is reported in Fig. 16. The simulations have been performed at a speed of
$V=50 \mathrm{~km} / \mathrm{h}\left(T_{I}=0, T_{F}=5 \mathrm{~s}\right)$ while the longitudinal deformations on the rail foot (the original one $\varepsilon_{x x 1}^{f r}$ and the approximate one $\varepsilon_{x x 1 a p}^{f r}$ ) have been measured on one single measure point $x_{m 1}=60.3 \mathrm{~m}$ without adding numerical disturbances (see Sect. 3.3). As it can be seen in the figure, the differences between the plotted quantities are quite negligible, confirming the accuracy of the quasi-linearity hypothesis. Quasilinearity hypothesis involves no mutual interactions between the elementary solutions associated to the fictitious loads. However, as shown by the results in Fig. 16, the passing of an axle is correctly influenced by the adiacent ones; this effect is mainly due to the overlapping among the adiacent traveling elementary solutions.

Despite its simplicity, the choice of the elementary model (employed to calculate the elementary functions needed for the axle load estimation, see Fig. 15) turnes out to be quite effective in approximating the real physical signal and then in estimating the real axle loads (thanks also to the LSO). The good behavior is confirmed by the experimental results (reported in this section and in Sect. 5.1), especially if we consider that they are produced by a real train in motion composed by three different wagons. 


\subsection{Least squares estimation}

Since the studied system is only approximatively linear, a least squares optimization (LSO) is needed to minimize the approximation errors between $\varepsilon_{x x k}^{f r}, \varepsilon_{x x k}^{s p}$ and $\varepsilon_{x x k a p}^{f r}, \varepsilon_{x x k a p}^{s p}$ and, at the same time, to optimize the values of $\widehat{N}_{i j}^{s i m}, \widehat{N}_{i j}^{s p}$. In this specific case linear not-weighted least squares have been considered [20-22].

To simulate the sampling due to the measurement process, the time domain $t \in\left[T_{I}, \bar{T}_{F}\right], \bar{T}_{F}=T_{F}-t_{11}$ (the shortest one among the domains $t \in\left[T_{I}, \hat{T}_{F}\right]$, $\hat{T}_{F}=T_{F}-t_{i j}$ ) has been discretized with the a sample time equal to $\Delta t=0.001 \mathrm{~s}$. Therefore both the simulated deformations $\varepsilon_{x x k}^{f r}\left(t_{h}\right)$ and the experimental ones $\varepsilon_{x x k}^{s p}\left(t_{h}\right)$ are known only at the times $t_{h}$ with $h=1,2, \ldots, N_{s}$ ( $N_{s}$ is the samples number while $t_{1}=T_{I}$ and $t_{N_{s}}=\bar{T}_{F}$ ); the same time discretization holds also for the fictitious deformations $\varepsilon_{x x f k i j}\left(t_{h}\right)$ employed to estimate $\varepsilon_{x x k}^{f r}, \varepsilon_{x x k}^{s p}$ (see Eqs. (16) and (17)). The sampled quantities can be written as

$\underline{\varepsilon}_{k}^{f r}=\left[\begin{array}{c}\varepsilon_{x x k}^{f r}\left(t_{1}\right) \\ \vdots \\ \varepsilon_{x x k}^{f r}\left(t_{h}\right) \\ \vdots \\ \varepsilon_{x x k}^{f r}\left(t_{N_{s}}\right)\end{array}\right] \in R^{N_{s}}$,

$A=\left[\begin{array}{c}\underline{\varepsilon}_{111} \cdots \underline{\varepsilon}_{1 n_{1} 1} \cdots \underline{\varepsilon}_{11 j} \cdots \underline{\varepsilon}_{1 n_{j} j} \cdots \underline{\varepsilon}_{11 n} \cdots \underline{\varepsilon}_{1 n_{n} n} \\ \vdots \\ \underline{\varepsilon}_{k 11} \cdots \underline{\varepsilon}_{k n_{1} 1} \cdots \underline{\varepsilon}_{k 1 j} \cdots \underline{\varepsilon}_{k n_{j} j} \cdots \underline{\varepsilon}_{k 1 n} \cdots \underline{\varepsilon}_{k n_{n} n} \\ \vdots \\ \underline{\varepsilon}_{N_{m} 11} \cdots \underline{\varepsilon}_{N_{m} n_{1} 1} \cdots \underline{\varepsilon}_{N_{m} 1 j} \cdots \underline{\varepsilon}_{N_{m} n_{j} j} \cdots \underline{\varepsilon}_{N_{m} 1 n} \cdots \underline{\varepsilon}_{N_{m} n_{n} n}\end{array}\right]$

$$
\begin{gathered}
\underline{\varepsilon}_{k}^{s p}=\left[\begin{array}{c}
\varepsilon_{x x k}^{s p}\left(t_{1}\right) \\
\vdots \\
\varepsilon_{x x k}^{s p}\left(t_{h}\right) \\
\vdots \\
\varepsilon_{x x k}^{s p}\left(t_{N_{s}}\right)
\end{array}\right] \in R^{N_{s}} \\
\underline{\varepsilon}_{k i j}=\left[\begin{array}{c}
\varepsilon_{x x f k i j}\left(t_{1}\right) \\
\vdots \\
\varepsilon_{x x f k i j}\left(t_{h}\right) \\
\vdots \\
\varepsilon_{x x f k i j}\left(t_{N_{s}}\right)
\end{array}\right] \in R^{N_{s}} .
\end{gathered}
$$

Taking into account the time sampling, Eqs. (16) and (17) become

$$
\begin{gathered}
\varepsilon_{x x k}^{f r}\left(t_{h}\right) \simeq \sum_{j=i}^{n} \sum_{i=1}^{n_{j}} \alpha_{i j}^{s i m} \varepsilon_{x x f k i j}\left(t_{h}\right) \\
h=1,2, \ldots, N_{s}, \quad k=1,2, \ldots, N_{m} \\
\varepsilon_{x x k}^{s p}\left(t_{h}\right) \simeq \sum_{j=i}^{n} \sum_{i=1}^{n_{j}} \alpha_{i j}^{s p} \varepsilon_{x x f k i j}\left(t_{h}\right) \\
h=1,2, \ldots, N_{s}, \quad k=1,2, \ldots, N_{m} .
\end{gathered}
$$

At this point, defining the matrix $A \in R^{N_{s} N_{m} \times n_{\text {tot }}}$ and the vectors $\underline{b}^{f r} \in R^{N_{s} N_{m}}, \underline{b}^{s p} \in R^{N_{s} N_{m}}$ as follows $\underline{b}^{f r}=\left[\begin{array}{c}\underline{\varepsilon}_{1}^{f r} \\ \vdots \\ \underline{\varepsilon}_{k}^{f r} \\ \vdots \\ \underline{\varepsilon}_{N_{m}}^{f r}\end{array}\right], \quad \underline{b}^{s p}=\left[\begin{array}{c}\underline{\varepsilon}_{1}^{s p} \\ \vdots \\ s p \\ \underline{\varepsilon}_{k} \\ \vdots \\ s p \\ \underline{\varepsilon}_{N_{m}}\end{array}\right]$

the matrix form of Eqs. (19) and (20) can be obtained:

$$
\begin{aligned}
& \underline{b}^{f r} \simeq A \underline{\alpha}^{s i m} \\
& \underline{b}^{s p} \simeq A \underline{\alpha}^{s p}
\end{aligned}
$$

where

$$
\begin{gathered}
\underline{\alpha}^{s p}=\left[\alpha_{11}^{s p} \ldots \alpha_{n_{1} 1}^{s p} \ldots \alpha_{1 j}^{s p} \ldots \alpha_{n_{j} j}^{s p} \ldots\right. \\
\left.\alpha_{1 n}^{s p} \ldots \alpha_{n_{n} n}^{s p}\right]^{T} \in R^{n_{t o t}}
\end{gathered}
$$




$$
\begin{aligned}
\underline{\alpha}^{s i m}= & {\left[\alpha_{11}^{s i m} \ldots \alpha_{n_{1} 1}^{s i m} \ldots \alpha_{1 j}^{s i m} \ldots \alpha_{n_{j} j}^{s i m} \ldots\right.} \\
& \left.\alpha_{1 n}^{s i m} \ldots \alpha_{n_{n} n}^{s i m}\right]^{T} \in R^{n_{t o t}} .
\end{aligned}
$$

By means of a least squares optimization (LQO) (in this case linear and not-weighted), it is now possible to minimize the squared 2-norms $E^{f r 2}=\left\|\underline{E}^{f r}\right\|_{2}^{2}$ and $E^{s p 2}=\left\|\underline{E}^{s p}\right\|_{2}^{2}$ of the approximation errors $\underline{E}^{f r}=A \underline{\alpha}^{s i m}-\underline{b}^{f r}, \underline{E}^{s p}=A \underline{\alpha}^{s p}-\underline{b}^{s p}$ present in Eqs. (23) and (24):

$$
\begin{aligned}
& \underline{\alpha}^{s i m}=\left(A^{T} A\right)^{-1} A^{T} \underline{b}^{f r} \\
& \underline{\alpha}^{s p}=\left(A^{T} A\right)^{-1} A^{T} \underline{b}^{s p}
\end{aligned}
$$

where the matrix $A^{T} A$ is invertible if and only if the rank of $A$ is maximum. Finally the values of the estimated vertical loads $\widehat{N}_{i j}^{s i m}, \widehat{N}_{i j}^{s p}$ can be evaluated starting from the knowledge of $\underline{\alpha}^{\text {sim }}$ and $\underline{\alpha}^{s p}$ :

$\underline{\widehat{N}}^{\text {sim }}=N_{f} \underline{\alpha}^{\text {sim }}$

$\underline{\widehat{N}}^{s p}=N_{f} \underline{\alpha}^{s p}$

in which

$$
\begin{aligned}
\underline{\widehat{N}}^{s i m}= & {\left[\widehat{N}_{11}^{s i m} \ldots \widehat{N}_{n_{1} 1}^{s i m} \ldots \widehat{N}_{1 j}^{s i m} \ldots \widehat{N}_{n_{j} j}^{s i m} \ldots\right.} \\
& \left.\widehat{N}_{1 n}^{s i m} \ldots \widehat{N}_{n_{n} n}^{s i m}\right]^{T} \in R^{n_{t o t}} \\
\underline{\widehat{N}}^{s p}= & {\left[\widehat{N}_{11}^{s p} \ldots \widehat{N}_{n_{1} 1}^{s p} \ldots \widehat{N}_{1 j}^{s p} \ldots \widehat{N}_{n_{j} j}^{s p} \ldots\right.} \\
& \left.\widehat{N}_{1 n}^{s p} \ldots \widehat{N}_{n_{n} n}^{s p}\right]^{T} \in R^{n_{t o t}} .
\end{aligned}
$$

\section{Performance of the WIM algorithm}

In this section the performance of the WIM algorithm for the estimation of the vertical axle loads $\widehat{N}$ starting from the longitudinal deformations $\varepsilon_{x x}$ on the rail foot will be tested. As previously said, the algorithm can use as input both the experimental quantities measured on the track and the simulated quantities calculated by the model of the railway track.

First of all the innovative WIM algorithm has been validated be means of the experimental data kindly provided by Ansaldo STS [19]. In this phase no disturbances have been considered because of the high quality of the experimental data and the low train speed $V$.
Subsequently the WIM algorithm has been tested with a suitable simulations campaign to verify the accuracy of the procedure when experimental data are not available; the whole physical model of the railway track has been developed and validated (see Sect. 3) just to provide the simulated inputs needed to test the WIM algorithm when there are no experimental data. In particular the attention will focus especially on the vehicle velocity $V$ and on the cut frequency $f_{n}$ of the physical system. In this second phase the measurement errors will play a fundamental role to evaluate the robustness of the WIM algorithm in any operating condition.

\subsection{WIM algorithm validation}

In order to validate the WIM algorithm, the experimental deformations on the rail foot $\varepsilon_{x x 1}^{s p}(t)=\varepsilon_{x x}^{s p}\left(x_{m 1}, t\right)$ and $\varepsilon_{x x 2}^{s p}(t)=\varepsilon_{x x}^{s p}\left(x_{m 2}, t\right)$ measured in two measure points $x_{m 1}=P_{I}=60.3 \mathrm{~m}$, $x_{m 2}=P_{F}=65.1 \mathrm{~m}$ (with $N_{m}=2$ ) have been considered. The experimental data have been compared with the analogous quantities $\varepsilon_{x x 1 a p}^{s p}(t)=\varepsilon_{x x a p}^{s p}\left(x_{m 1}, t\right)$, $\varepsilon_{x x 2 a p}^{s p}(t)=\varepsilon_{x x a p}^{s p}\left(x_{m 2}, t\right)$ estimated by means of the WIM algorithm (see Eq. (17)) (in the benchmark case $V=15.4 \mathrm{~km} / \mathrm{h}, T_{I}=0 \mathrm{~s}, T_{F}=15.3 \mathrm{~s}$ ).

The comparison between the measured deformations $\varepsilon_{x x 1}^{s p}, \varepsilon_{x x 2}^{s p}$ and the estimated ones $\varepsilon_{x x 1 a p}^{s p}, \varepsilon_{x x 2 a p}^{s p}$ in the measure points $x_{m 1}, x_{m 2}$ are represented in Figs. 17 and 18 (see also Fig. 14).

As visible in the figures, the comparison shows a good agreement between experimental and estimated quantities and confirms the accuracy of the WIM procedure. The choice of the elementary model (employed to calculate the elementary functions needed for the axle load estimation, see Fig. 15) turned out to be quite effective in approximating the real physical signal and then in estimating the real axle loads (thanks also to the LSO). Furthermore it is worth noting that the considered experimental results (reported in this section and in Sect. 4.2) are produced by a real train in motion composed by three different wagons. The values of the nominal and estimated vertical loads on the vehicle wheels $N_{i j}, \widehat{N}_{i j}^{s p}$ are summarized in Table 10 together with the relative errors $e_{i j}^{s p}=\frac{\widehat{N}_{i j}^{s p}-N_{i j}}{N_{i j}}$.

The algorithm accuracy in estimating the vertical loads (relative errors equal to $1-2 \%$ ) is mainly due to the capability of correctly describing the global shape 


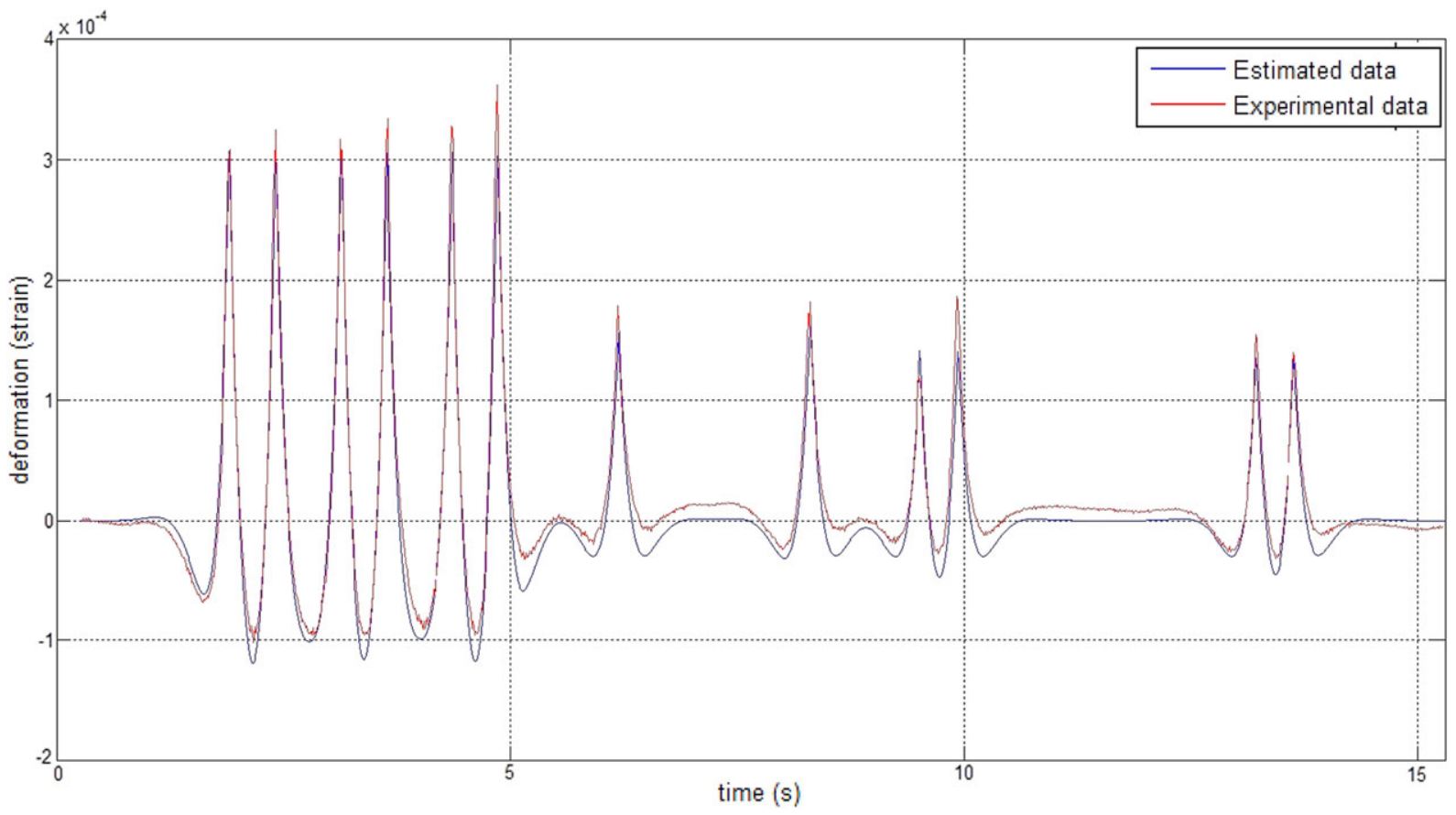

Fig. 17 Measured and estimated longitudinal deformations $\varepsilon_{x x 1}^{s p}$ and $\varepsilon_{x x 1 a p}^{s p}$ in the measure point $x_{m 1}=60.3 \mathrm{~m}$

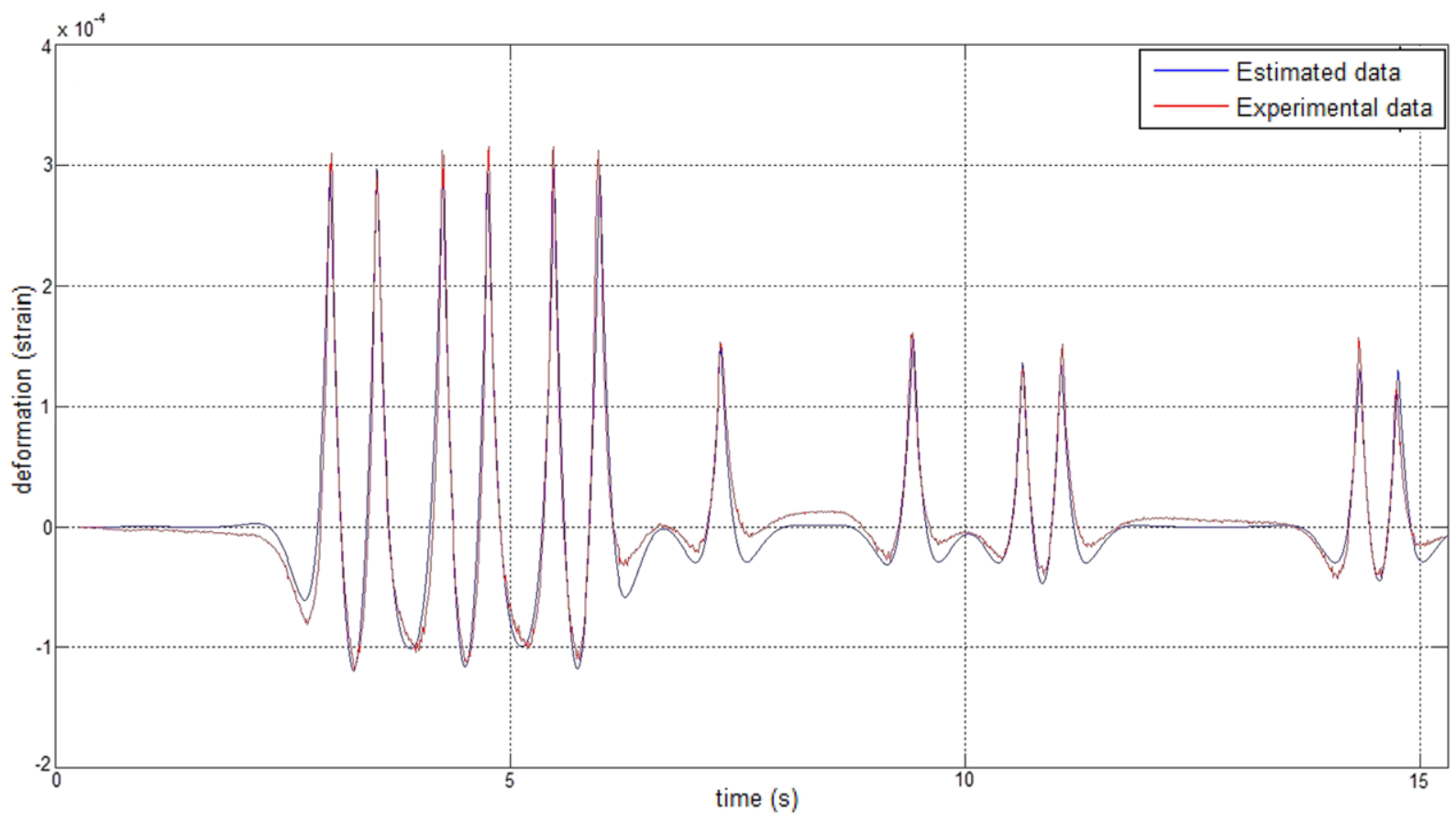

Fig. 18 Measured and estimated longitudinal deformations $\varepsilon_{x x 2}^{s p}$ and $\varepsilon_{x x 2 a p}^{s p}$ in the measure point $x_{m 2}=65.1 \mathrm{~m}$ 
Table 10 Nominal and estimated vertical loads on the vehicle wheels $N_{i j}, \widehat{N}_{i j}^{s p}$

\begin{tabular}{lllllllll}
\hline Parameter & Units & Value & Parameter & Units & Value & Parameter & Units & Value \\
\hline$N_{11}$ & $\mathrm{~N}$ & 86655 & $\widehat{N}_{11}^{s p}$ & $\mathrm{~N}$ & 86707 & $e_{11}^{s p}$ & - & $+0.1 \%$ \\
$N_{21}$ & $\mathrm{~N}$ & 86655 & $\widehat{N}_{21}^{s p}$ & $\mathrm{~N}$ & 86717 & $e_{21}^{s p}$ & - & $+0.1 \%$ \\
$N_{31}$ & $\mathrm{~N}$ & 86655 & $\widehat{N}_{11}^{s p}$ & $\mathrm{~N}$ & 86699 & $e_{31}^{s p}$ & - & $+0.1 \%$ \\
$N_{41}$ & $\mathrm{~N}$ & 86655 & $\widehat{N}_{41}^{s p}$ & $\mathrm{~N}$ & 86701 & $e_{41}^{s p}$ & - & $+0.1 \%$ \\
$N_{51}$ & $\mathrm{~N}$ & 86655 & $\widehat{N}_{11}^{s p}$ & $\mathrm{~N}$ & 86710 & $e_{51}^{s p}$ & - & $+0.1 \%$ \\
$N_{61}$ & $\mathrm{~N}$ & 86655 & $\widehat{N}_{11}^{s p}$ & $\mathrm{~N}$ & 86712 & $e_{61}^{s p}$ & - & $+0.1 \%$ \\
$N_{12}$ & $\mathrm{~N}$ & 39240 & $\widehat{N}_{12}^{s p}$ & $\mathrm{~N}$ & 38093 & $e_{12}^{s p}$ & - & $-2.9 \%$ \\
$N_{22}$ & $\mathrm{~N}$ & 39240 & $\widehat{N}_{22}^{s p}$ & $\mathrm{~N}$ & 39407 & $e_{22}^{s p}$ & - & $+0.4 \%$ \\
$N_{13}$ & $\mathrm{~N}$ & 38014 & $\widehat{N}_{13}^{s p}$ & $\mathrm{~N}$ & 39061 & $e_{13}^{s p}$ & - & $+2.8 \%$ \\
$N_{23}$ & $\mathrm{~N}$ & 38014 & $\widehat{N}_{23}^{s p}$ & $\mathrm{~N}$ & 39070 & $e_{23}^{s p}$ & - & $+2.8 \%$ \\
$N_{33}$ & $\mathrm{~N}$ & 38014 & $\widehat{N}_{33}^{s p}$ & $\mathrm{~N}$ & 37519 & $e_{33}^{s p}$ & - & $-1.3 \%$ \\
$N_{43}$ & $\mathrm{~N}$ & 38014 & $\widehat{N}_{43}^{s p}$ & $\mathrm{~N}$ & 37533 & $e_{43}^{s p}$ & - & $-1.3 \%$ \\
\hline
\end{tabular}

of the solutions (both in the space and in the time) despite the errors in terms of peak values (sometimes greater than $10 \%$ ). These peak errors are caused by not modeled dynamics and disturbances and by the cross-effects between adjacent axles present in particular operating conditions involving low distances between the axles and high train speeds; in fact, since the system is only approximately linear, the cross-effect cannot be completely overcome.

The good description of the global solution shape (less affected by errors on the peak values) is the most interesting feature of the proposed algorithm and it is possible because the new procedure is based on a Least Squares approach taking into account the whole shape of the solutions instead of its peak values. Moreover the considered strategy further reduces the bandwidth required by the algorithm to correctly estimate the vertical loads on the wheels.

The values of the main parameters of the ODE integrator like the maximum step size MaxStep, the absolute and relative tolerances AbsTol, RelTol and the maximum dimension $h_{\max }$ of the elements used in the FEM discretization of the rail beam are the same reported in Table 7 [20-22].

\subsection{The numerical simulations campaign}

In this section the longitudinal deformations $\varepsilon_{x x k}^{f r}(t)=\varepsilon_{x x}^{f r}\left(x_{m k}, t\right)$ evaluated through the physical model of the railway track (see Sect. 3) are compared with the deformations $\varepsilon_{x x k a p}^{f r}(t)=\varepsilon_{x x a p}^{f r}\left(x_{m k}, t\right)$ estimated by means of the WIM algorithm (see Eq. (16)).

The comparison between the deformations calculated by the physical model and those estimated by the WIM procedure is quite important to test the algorithm accuracy when experimental data are not available. Furthermore in this case also the measurement errors will be considered (according to Sect. 3.3) in order to evaluate the algorithm robustness in presence of disturbances.

To perform the comparison between simulated and estimated deformations $\left(\varepsilon_{x x k}^{f r}, \varepsilon_{x x k a p}^{f r}\right.$ respectively), an extensive simulations campaign has been carried out. In particular in this work the dependence of the relative errors $e_{i j}^{s i m}=\frac{\widehat{N}_{i j}^{s i m}-N_{i j}}{N_{i j}}$ on the vehicle speed $V$ and the cut frequency $f_{n}$ of the physical system is analysed. In Table 11 the considered variation ranges for the previous quantities are reported together with the resolutions adopted for the range discretization $(\Delta V$, $\Delta f_{n}$ respectively); the range boundaries take into account both the usual traveling velocity of the freight wagons and at the same time the typical cut frequency of the studied physical system and measurement chain.

The global performance of the WIM algorithm have been studied by considering the maximum relative error $e_{\max }^{\operatorname{sim}}\left(V, f_{n}\right)$ :

$e_{\max }^{s i m}=\left\|\underline{e}^{s i m}\right\|_{\infty}=\max _{1 \leq i \leq n_{j}, 1 \leq j \leq n}\left|e_{i j}^{s i m}\right|$ 
Table 11 Variation ranges of $V$ and $f_{n}$ adopted for the simulations campaign and their discretization

\begin{tabular}{|c|c|c|c|c|c|}
\hline Velocity & Unit & Value & Cut frequency & Units & Value \\
\hline Min. train velocity $V_{\min }$ & $\mathrm{m} / \mathrm{s}$ & 10 & Min cut off freq. $f_{n \text { min }}$ & $\mathrm{Hz}$ & 10 \\
\hline Max. train velocity $V_{\max }$ & $\mathrm{m} / \mathrm{s}$ & 40 & Max cut off freq. $f_{n \max }$ & $\mathrm{Hz}$ & 40 \\
\hline Sim. number $N_{v}$ & - & 100 & Sim. number $N_{f_{n}}$ & - & 100 \\
\hline $\begin{array}{l}\Delta V=\left(V_{\max }-V_{\min }\right) /\left(N_{v}-1\right), \\
V_{I}=V_{\min }+(I-1) \Delta V, \\
I=1, \ldots, N_{v}\end{array}$ & $\mathrm{~m} / \mathrm{s}$ & 0.303 & $\begin{array}{l}\Delta f_{n}=\left(f_{n \max }-f_{n \min }\right) /\left(N_{f_{n}}-1\right) \\
f_{n J}=f_{n \min }+(J-1) \Delta f_{n} \\
J=1, \ldots, N_{f_{n}}\end{array}$ & $\mathrm{~Hz}$ & 0.303 \\
\hline
\end{tabular}

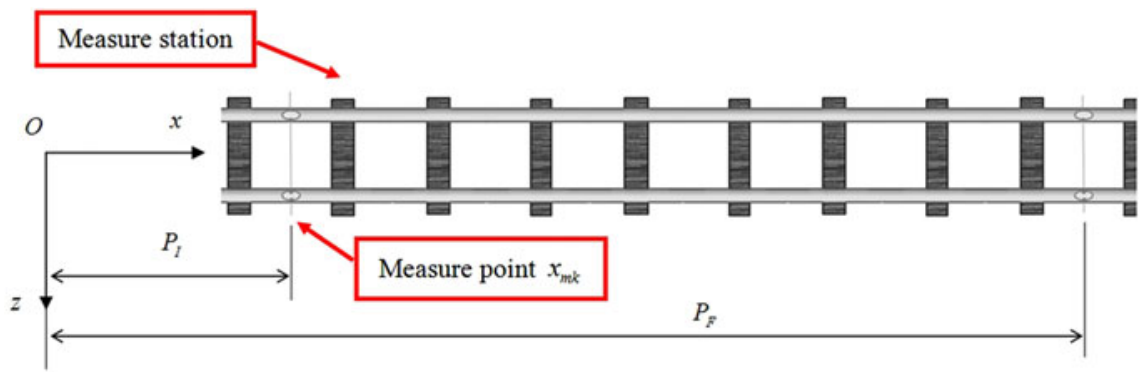

(a) First measure station layout: two measure points $x_{m 1}, x_{m 2}$

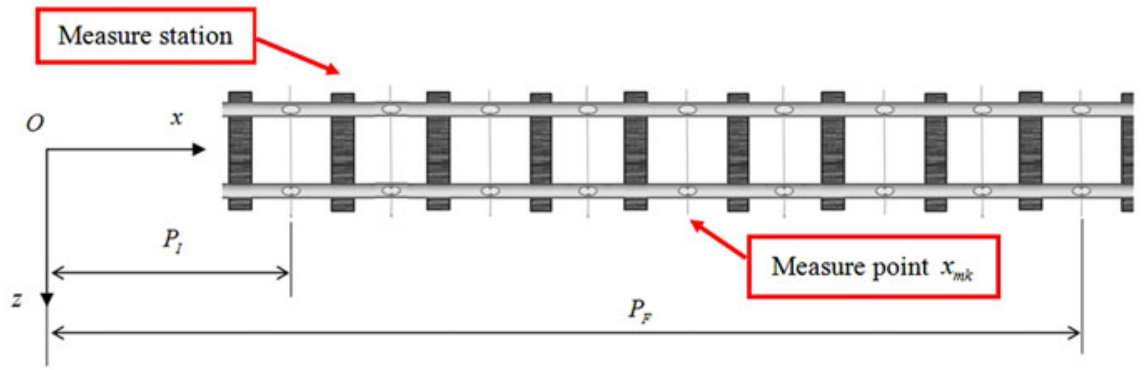

(b) Second measure station layout: nine measure points $x_{m 1}, \ldots x_{m 9}$

Fig. 19 Comparison between the considered measure station layouts

where

$$
\begin{gathered}
\underline{e}^{s i m}=\left[e_{11}^{s i m} \ldots e_{n_{1} 1}^{s i m} \ldots e_{1 j}^{s i m} \ldots e_{n_{j} j}^{s i m} \ldots\right. \\
\left.e_{1 n}^{s i m} \ldots e_{n_{n} n}^{s i m}\right]^{T} \in R^{n_{t o t}}
\end{gathered}
$$

and analysing the behaviour of the error surface $e_{\max }^{\operatorname{sim}}\left(V, f_{n}\right)$.

Two different layouts of measure station with a different number $N_{m}$ of measure points and distance $l_{k}$ between two consecutive points (as visible in Fig. 19) are taken into account with the aim of investigating the error sensibility with respect to the choice of the measure points. Table 12 summarizes the measure point positions $x_{m k}$ of each measure station layout together with the values of $N_{m}$ and $l_{k}$ (the initial and final points of the measure station are always $P_{I}=60.3$, $\left.P_{F}=65.1\right)$.

The error surfaces $e_{\max }^{s i m}\left(V, f_{n}\right)$ relative to the two different measure station layouts (2 and 9 measure points on the railway track) are illustrated in Figs. 20 and 21. In particular for all the studied cases both a three-dimensional and a two-dimensional (from the top, parallel to the plane $V-f_{n}$ ) view of the surface $e_{\max }^{s i m}\left(V, f_{n}\right)$ are reported to better highlight the error behaviour.

The numerical noise present on the error surfaces visible in the previous figures is mainly due to disturbances characterising the physical model of the railway tracks and always different in each numerical sim- 
Table 12 Measure point positions $x_{m k}$, total number of points $N_{m}$ and distance $l_{k}$ between two consecutive points of the considered measure station layouts

\begin{tabular}{llll}
\hline Meas. st. layout & Points $N_{m}$ & Points distance $l_{k}(\mathrm{~m})$ & Meas. point positions $x_{m k}(\mathrm{~m})$ \\
\hline 1st meas. layout & 2 & 4.8 & $x_{m 1}=60.3, x_{m 2}=65.1$ \\
3rd meas. layout & 9 & 0.6 & $x_{m 1}=60.3, x_{m 2}=60.9, \ldots, x_{m 8}=64.5, x_{m 9}=65.1$ \\
\hline
\end{tabular}

ulation (see Sect. 3.3). For the sake of clearness, for all the studied measure station layout, the domain of the plane $V-f_{n}$ where the WIM algorithm shows a better accuracy is highlighted (see Figs. 20 and 21). By convention the accuracy zone is defined as the domain where the error $e_{\max }^{\operatorname{sim}}\left(V, f_{n}\right)$ is minor or equal to 0.1 .

As it can be seen in the previous figures, all the considered measure station layouts show a quite large accuracy zone even for relatively low values of $f_{n}$ and relatively high values of $V$ (always with respect to the range of velocities $V$ and cut frequencies $f_{n}$ considered for freight trains). Moreover an increase of the number $N_{m}$ of measure points $x_{m k}$ on the railway track leads to a better accuracy of the WIM algorithm and consequently to an enlargement of the accuracy zone (see Figs. 20 and 21). The improvement of the WIM algorithm performance is mainly due to the better accuracy of the least squares optimization (LSO), on which the algorithm is based, obtainable with an higher number of measure points. Moreover, with more measure points, the global shape of the signal can be better reproduced by the algorithm inside the measure station. At the same time an higher number of measure points increases the dimensions of the measure station and involves higher economical costs; therefore a compromise between these two aspects is always needed. The values of the main parameters of the ODE integrator like the maximum step size MaxStep, the absolute and relative tolerances AbsTol, RelTol and the maximum dimension $h_{\max }$ of the elements used in the FEM discretization of the rail beam are always the same reported in Table 7 [20-22].

\section{Conclusions and further developments}

In this paper the authors presented an innovative WIM algorithm with the aim of estimating the vertical axle loads $\widehat{N}$ of railway vehicles. The algorithm is tested by assuming as input a perturbed and bandwidth limited rail bending measurements (performed through strain sensitive elements used to evaluate the longitudinal deformations $\varepsilon_{x x}$ on the rail foot) in order to verify as much as possible the robustness of the proposed estimation algorithm. In order to estimate the axle loads, the algorithm tries to approximate the measured physical input through a set of elementary functions. The elementary functions are evaluated by simulating a single fictitious load moving on the track (see Fig. 15). This elementary model is very simple and depends on few significant parameters; in this way it can be easily tuned, for example, by performing the estimation on a benchmark known train travelling through the measure station. In particular the simple model employed for the basis construction cannot depend on the vehicle geometrical and physical parameters since they are obviously unknown. The measured signal is then approximated by means of Least Square Optimization (LSO) techniques starting from the set of elementary functions: more particularly, the measured signal is rebuilt through a linear combination of the elementary functions, the coefficients of which are the axle loads to be estimated.

The authors have also developed a physical model of the railway track; the model consists of the planar FEM model of the infrastructure and of the twodimensional multibody model of the vehicle (the effects of lateral dynamics are treated as disturbances) and naturally considers the coupling between the adjacent loads moving on the track and the vehicle dynamics. The physical model of the track and the innovative WIM algorithm (both taking into account possible measurement errors) have been validated be means of the experimental data kindly provided by Ansaldo STS [19] and have been implemented in the Matlab and Comsol Multiphysics environments [8, 18]. In particular the model of the railway track has been developed expressly to test the WIM algorithm with a suitable simulation campaign when experimental data are not available and provides synthetic simulated inputs to test the WIM algorithm in absence of experimental inputs. Obviously this model is not used inside the 


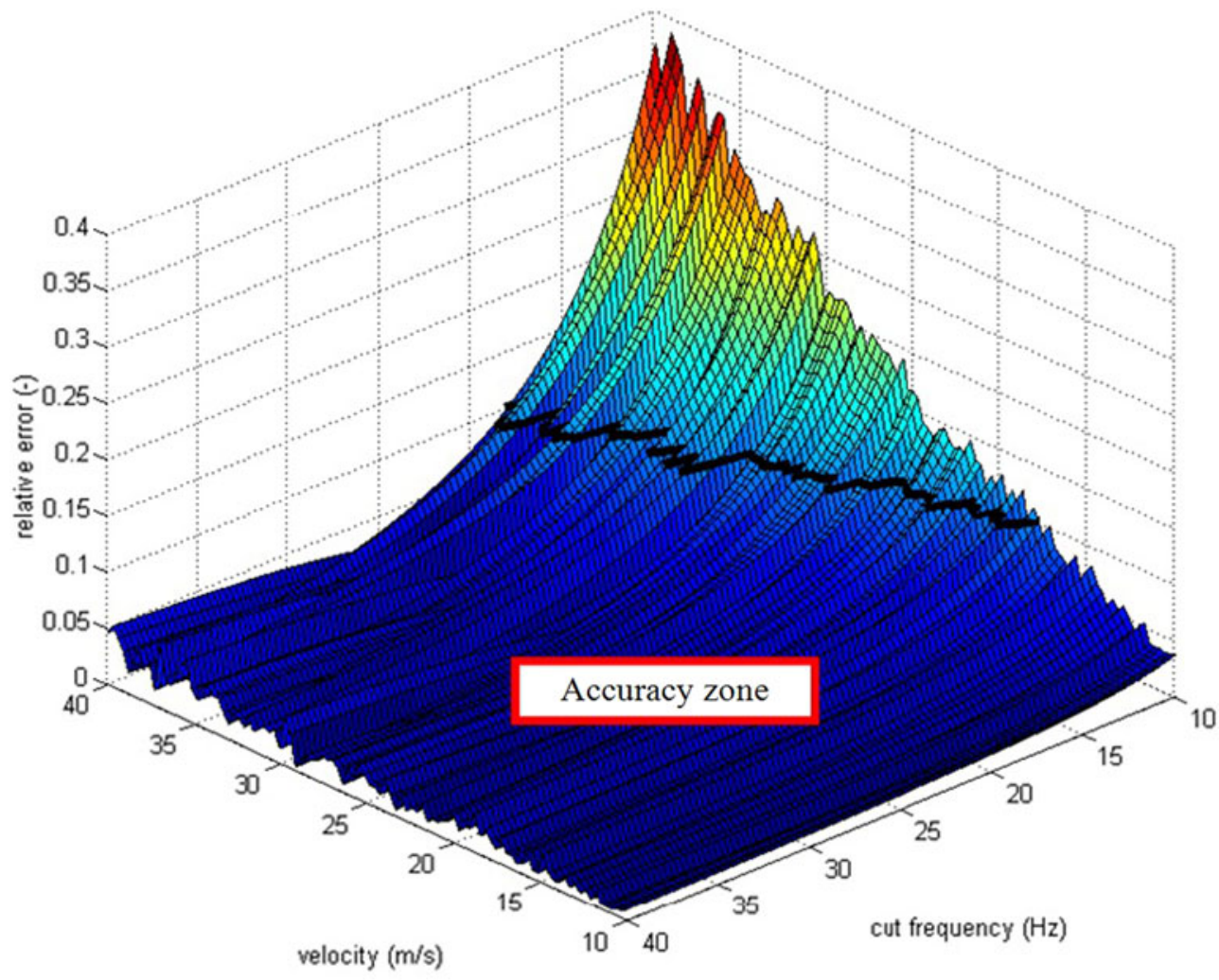

(a) Error surface: three-dimensional view

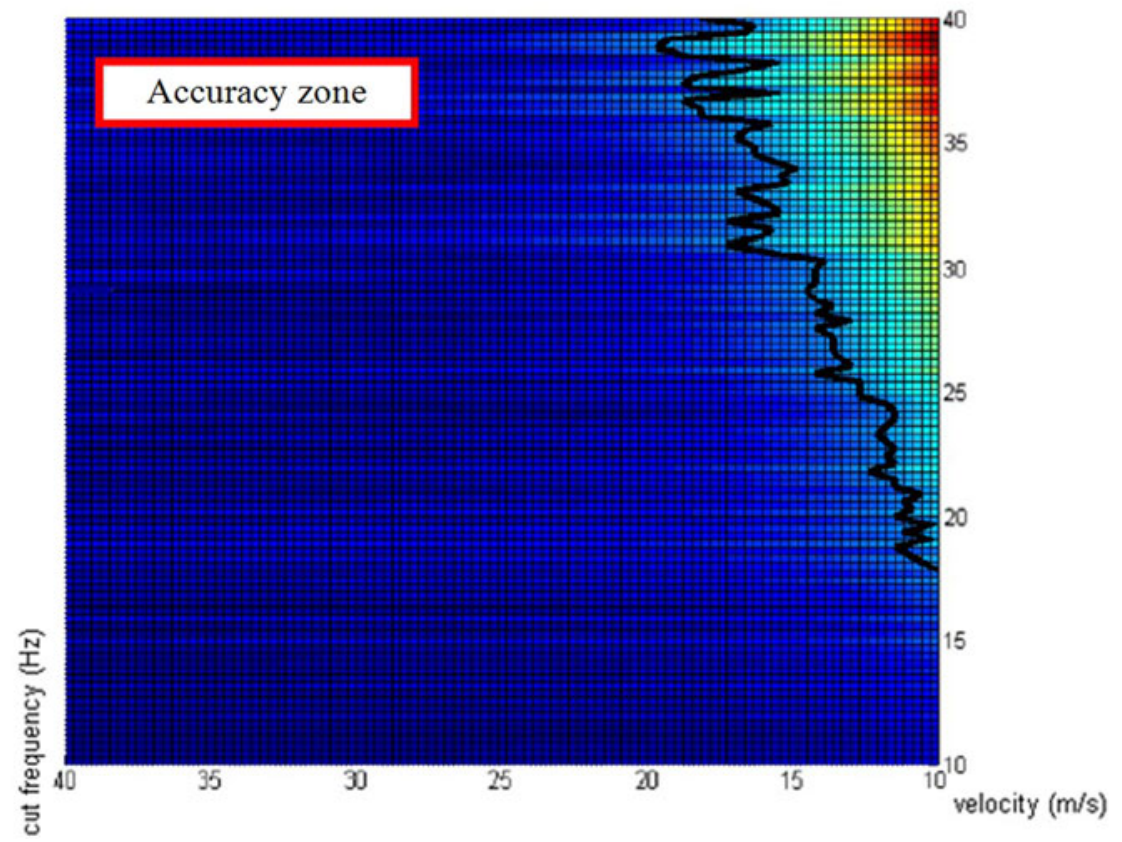

(b) Error surface: two-dimensional view

Fig. 20 Error surface for the first measure layout: two measure points $x_{m 1}=60.3 \mathrm{~m}, x_{m 2}=65.1 \mathrm{~m}$ 


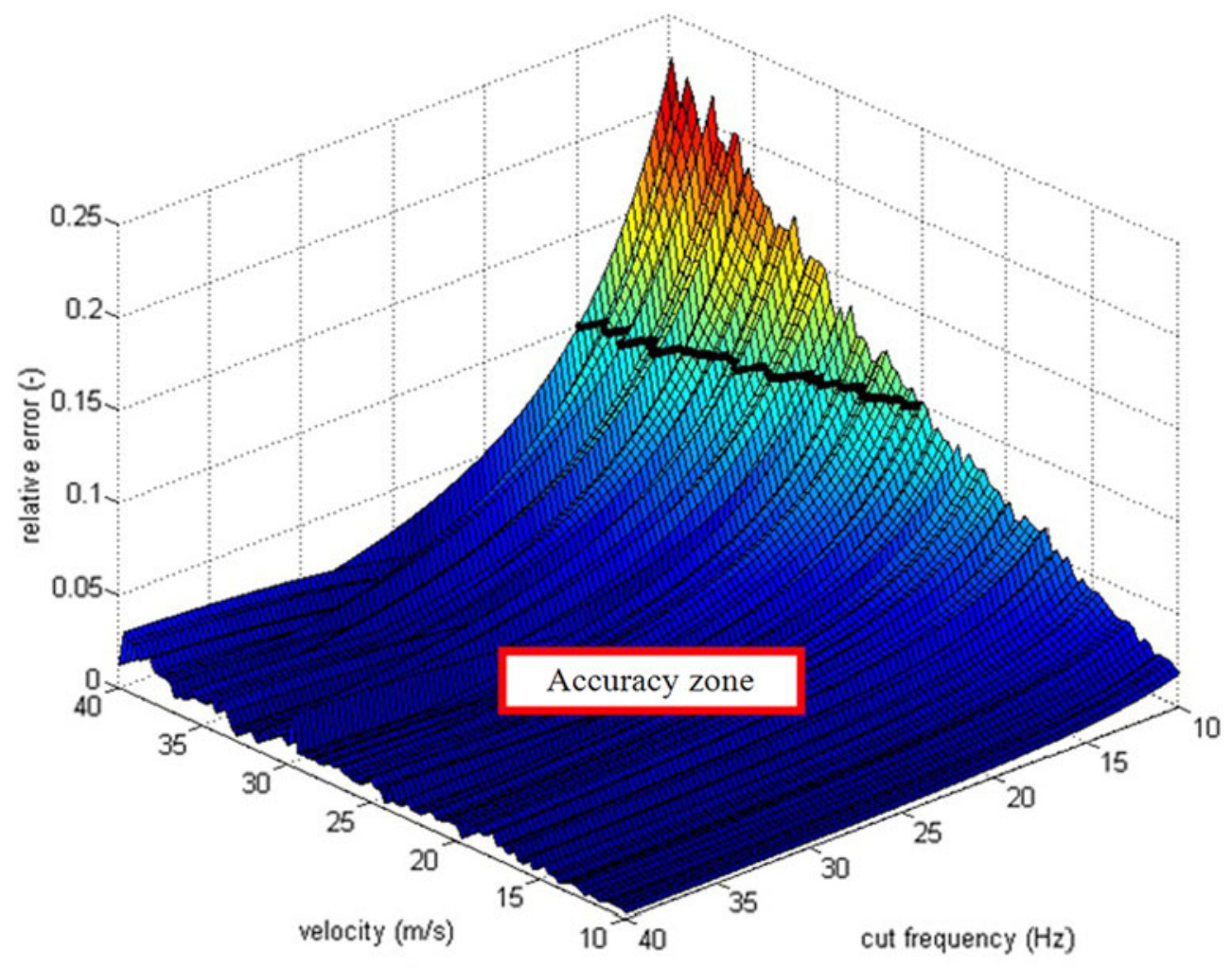

(a) Error surface: three-dimensional view

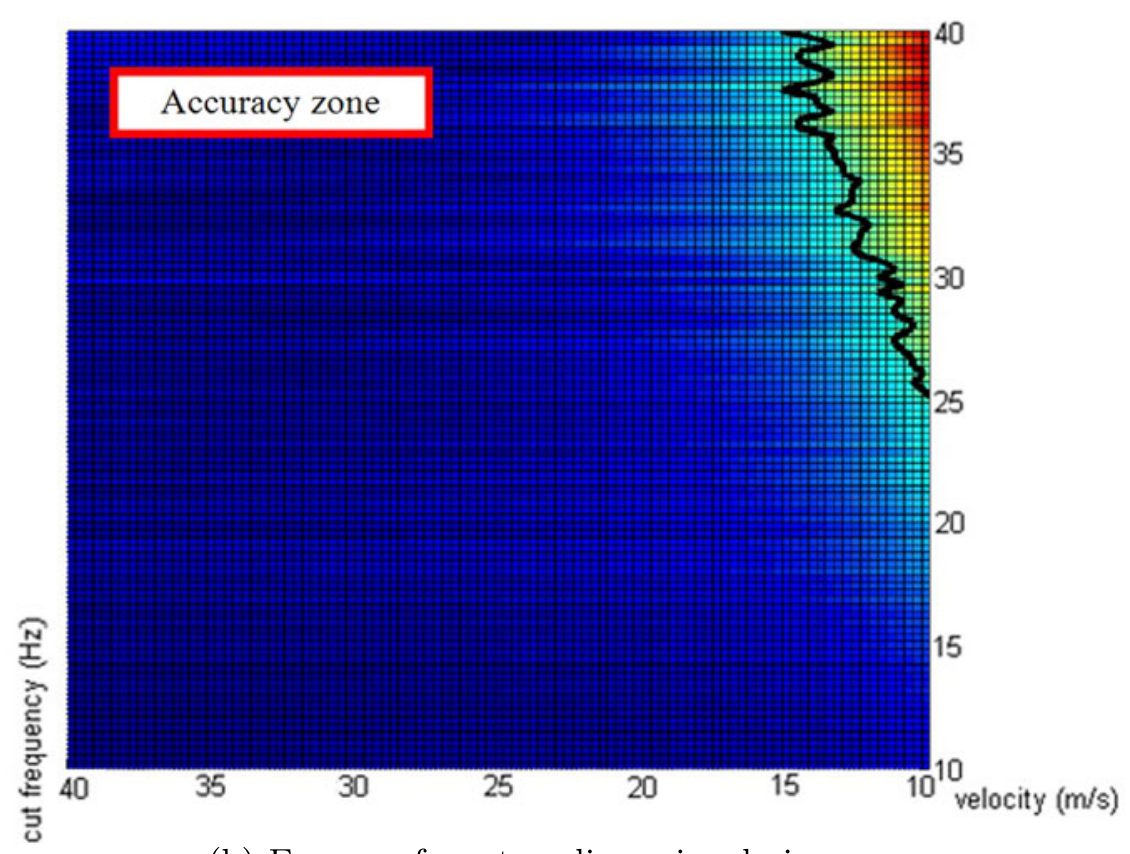

(b) Error surface: two-dimensional view

Fig. 21 Error surface for the second measure layout: nine measure points $x_{m 1}=60.3 \mathrm{~m}, x_{m 2}=60.9 \mathrm{~m}, \ldots, x_{m 8}=64.5 \mathrm{~m}$, $x_{m 9}=65.1 \mathrm{~m}$ 
WIM algorithm because the geometrical and physical train parameters are unknown.

The validation of the new WIM algorithm (see Sect. 5.1 on the WIM algorithm validation) highlighted a good agreement between the estimated quantities and the experimental data, confirming the good accuracy of the procedure. At the same time the comparison between the data obtained by means of the physical model of the railway track and those estimated by the WIM algorithm (see Sect. 5.2 on the numerical simulations campaign) showed a good accuracy and robustness of the estimation procedure in any operating condition (within the whole range of velocities $V$ and cut frequencies $f_{n}$ considered for freight trains).

Concerning the future developments, the improvements will regard first of all the WIM algorithm. In particular other estimation procedures (like weighted least square optimization (WLSO) and nonlinear least square optimization (NLSO)) and other possible physical inputs of the algorithm besides the longitudinal deformations $\varepsilon_{x x}$ (like generic stresses $\sigma$ and deformations $\varepsilon$ and the forces on the sleepers $N_{S}$ as well as a combination of such physical quantities) will be considered for estimating the vertical axle loads $\widehat{N}$.

As regards the new physical inputs, also inertial measurements will be taken into account to further increase the bandwidth of the system and to identify specific defects of the rolling surfaces like wheelflats. At the same time radio frequency identification techniques employed to identify the main characteristics of the train composition [23].

The goal of the improved algorithms will be also the estimation of other geometrical and physical characteristics of the railway vehicle besides the vertical axle loads like center of mass positions, masses and inertial tensors of the wagons.

From an experimental point of view further experimental tests are scheduled for the future by Ansaldo STS. The new experimental data will concern wagons travelling at higher speeds than that considered in the paper, wagons characterized by different geometries and wagons subjected to unbalanced loads (both in longitudinal and in lateral direction). Moreover the authors are currently waiting for receiving from Ansaldo STS more extensive experimental data concerning measurement campaigns performed on different railway tracks with different measurement layouts and different measured physical quantities as inputs of the WIM algorithm.
Finally a real prototype of the measure station is being currently designed and will be soon assembled on a suitable railway track. The prototype will aim to test the accuracy and the robustness of the WIM algorithms together with the effectiveness of the various measure station layouts.

Acknowledgements Authors would like to thank Eng. Nadia Mazzino and Eng. Giuseppe Parente of Ansaldo STS for providing and giving the permission to edit the experimental data needed for the validation of both the physical model of the railway track and the new WIM algorithm.

\section{References}

1. Bracciali A, Ciuffi R, Piccioli F (2001) Progetto e validazione di un sensore estensimetrico multifunzionale per il binario ferroviario. In: Proceedings of XXX congresso AIAS, Alghero, Italy

2. Del Prete C, Rosso C (2009) An easy instrument and a methodology for the monitoring and the diagnosis of a rail. Mech Syst Signal Process 23(3):940-956

3. (2012) Official site of Kistler. http://www.kistler.com

4. (2012). Official site of Tagmaster. http://www.tagmaster. com

5. Kolakowski P, Sekula K (2010) Piezo-based weigh-inmotion system for the railway transport. Struct Control Health Monit. doi:10.1002/stc.416

6. (2012) Official site of Stock Equipment Company. http:// www.stockequipment.com

7. (2012) Official site of Cardinal. http://www.cardinalscale. com

8. (2012) Official site of The MathWorks. http://www. mathworks.com

9. Iwnicki S (2006) Handbook of railway vehicle dynamics. Taylor \& Francis, London

10. Esveld C (2001) Modern railway track. Delft University of Technology, Delft

11. Kisilowski J, Knothe K (1991) Advanced railway vehiclesystem dynamics. Wydawnictwa Naukowo-Techniczne, Warsaw

12. Bracciali A, Ciuffi R, Piccioli F (2001) Soluzione numerica del modello PDE della dinamica verticale di un binario. In: Proceedings of XXX congresso AIAS, Alghero, Italy

13. Dahlberg T (2001) Some railroad settlement models-a critical review. Proc Inst Mech Eng, F J Rail Rapid Transit 215:289-300

14. Senyansky DM (2003) Problem of increasing the accuracy of railway carriages weighing in motion. In: VII IMEKO world Congress on metrology in the 3rd millennium, Dubrovnik, Croatia

15. Da Costa Marques Pimentel RM, Beirão Barbosa MC, Silva Costa NM, Ferreira Ribeiro DR, De Almeida Ferreira LA, Moita Araújo FM, Bártolo Calçada RA (2008) Hybrid fiber-optic/electrical measurement system for characterization of railway traffic and its effects on a short span bridge. IEEE Sens J 8(7):289-300 
16. Bracciali A, Piccioli F (2001) Attenuation of rail vibration: analysis of experimental data. In: Proceedings of WCRR 2001 World Congress on Railway Research, Köhln, Germany

17. Zakeri JA, Xia H, Fan JJ (2009) Dynamic responses of train-track system to single rail irregularity. Lat Am J Solids Struct 6(2):183-204

18. (2012) Official site of Comsol Multiphysics. http://www. comsol.com

19. Mazzino N, Parente G (2012) Dati sperimentali per la verifica preliminare di algoritmi WIM per la pesatura dinamica. In: Internal technical documentation Ansaldo STS, Genova, Italia

20. Shampine LF, Reichelt MW (1997) The Matlab ODE suite. SIAM J Sci Comput 18:1-22
21. Kelley C (1995) Iterative methods for linear and nonlinear equations. SIAM, Philadelphia

22. Nocedal J, Wright S (1999) Numerical optimization. Springer series in operation. Research, Berlin

23. Garcia JCY, Garcia YSM, De Gracia Santos J (2009) TransID: automatic ID and data capture for rail freight asset management. IEEE Internet Comput 13(1):22-30

24. Ferreira AJM, Castro LMS, Bertoluzza S (2011) Analysis of plates on Winkler foundation by wavelet collocation. Meccanica 46(4):865-873

25. Zenkour AM, Radwan AF (2012) On the simple and mixed first-order theories for functionally graded plates resting on elastic foundations. Meccanica. doi:10.1007/s11012-0129680-9 